\title{
Volume Weighted Measures of Eternal Inflation in the Bousso-Polchinski Landscape
}

\author{
Timothy Clifton ${ }^{1}$, Stephen Shenker ${ }^{2}$ and Navin Sivanandam ${ }^{3}$ \\ Department of Physics, Stanford University, CA 94305, USA
}

\begin{abstract}
We consider the cosmological dynamics associated with volume weighted measures of eternal inflation, in the Bousso-Polchinski model of the string theory landscape. We find that this measure predicts that observers are most likely to find themselves in low energy vacua with one flux considerably larger than the rest. Furthermore, it allows for a satisfactory anthropic explanation of the cosmological constant problem by producing a smooth, and approximately constant, distribution of potentially observable values of $\Lambda$. The low energy vacua selected by this measure are often short lived. If we require anthropically acceptable vacua to have a minimum life-time of 10 billion years, then for reasonable parameters a typical observer should expect their vacuum to have a life-time of approximately 12 billion years. This prediction is model dependent, but may point toward a solution to the coincidence problem of cosmology.
\end{abstract}

\footnotetext{
${ }^{1}$ T.Clifton@cantab.net

${ }^{2}$ SShenker@stanford.edu

${ }^{3}$ NavinS@stanford.edu
} 


\section{Contents}

1 Introduction $\quad 2$

2 The Model

2.1 Volume Weighting ......................... . . 4

2.2 The Bousso-Polchinski Landscape and Transitions . . . . . . . . . . . . . 6

2.3 Anthropic Considerations . . . . . . . . . . . . . . . . . 9

2.3.1 Cosmological constant bound .................... 9

2.3 .2 Life-time bound . . . . . . . . . . . . . . . 10

3 Populating at High Energies $\quad 11$

4 Tunneling from High to Low Energies $\quad 13$

4.1 Expectation Values . . . . . . . . . . . . . . . . 16

4.2 The Single Peak Approximation . . . . . . . . . . . . . . . . 17

4.3 Analytic Approximations . . . . . . . . . . . . . . . . 18

5 Transitions into the Anthropic Shell $\quad 20$

5.1 Peaking From the Last Step . . . . . . . . . . . . . . . . 21

6 The Most Probable Vacua $\quad 22$

6.1 Peaking About the Most Likely Point . . . . . . . . . . . . . . 23

6.2 Pressure Against the Life-Time Boundary . . . . . . . . . . . . . 24

\begin{tabular}{llr}
7 & Discussion & 25 \\
\hline
\end{tabular}

$\begin{array}{ll}\text { A Changing the Location of the High-Energy Barrier } & 28\end{array}$

B Expectation Values Due to Tunneling From High Energy

C The Single Peak Approximation for the Final Transition 31 


\section{Introduction}

There is now strong evidence that string theory contains a large number of metastable vacua, collectively referred to as the landscape [1]. In order to make progress in understanding the place of a typical observer in this landscape of possibilities we must understand which vacua are possible, and the method of their population. The former of these problems is a current topic of research and we will not attempt to contribute to this field here. Instead we will take the pioneering model of Bousso and Polchinski [2], and investigate the latter.

The population mechanism for the landscape is eternal inflation. De Sitter vacua are constantly expanding and, in string theory, are meta-stable. As these vacua expand they nucleate bubbles of new vacuum, which themselves expand and nucleate further bubbles. In this way it is expected that all corners of the landscape are realised 4 , and the pertinent question is then which are the most probable. The fundamentals involved in answering this question are currently a matter of some contention: A difficulty often referred to as 'the measure problem.'

Various different measures have been proposed in the literature. We will not attempt to describe them all here, nor compare their various implicit benefits and difficulties. Instead, we will take one particular class of measures, and investigate the outcome of using them to predict the likely vacua that observers will find themselves in. The class we will study are the volume weighted measures, proposed by Linde [4. This class embodies the appealing intuition of rewarding the fastest expanding vacua. A refinement of this measure has recently been shown to produce results with surprisingly little dependence on the choice of time parameterization, and that do not exhibit a hotness (or youngness) paradox [5]. Using the Bousso-Polchinski landscape to investigate the results of volume-weighted eternal inflation has the advantage of this model already having been used to investigate other measures, namely the bubble-counting measure of [6, 7] and the holographic measure of [8, 9]. This will allow us to directly compare the results of several candidate measures within the same theoretical framework.

The Bousso-Polchinski landscape has inherent in it a number of approximations. For example, in this toy model the charges and their associated moduli are independent of fluxes, which is unlikely to be true of the real landscape. The contribution of slow-roll inflation is also ignored. Within the Bousso-Polchinski framework we will make several further approximations, for analytic convenience, such as treating discrete fluxes as continuous variables. Nonetheless we will be able to find, within this model, reliable predictions for the distribution of the cosmological constant, the likely configuration of fluxes and even the typical lifetime of a 'pocket universe'.

In section 2 we will give a brief account of volume weighted eternal inflation and, using examples, will give an account of how to calculate probabilities in this measure. We will follow this with a description of the Bousso-Polchinski landscape, and the transition rates expected within it. We will then reflect on some anthropic considerations that can be applied to this landscape. In particular, we consider anthropic bounds on the cosmological constant and on

\footnotetext{
${ }^{4}$ For a recent study of possible mechanisms to divide the landscape into different regions see 3 .
} 
the lifetime of vacua that must be obeyed if observers are to be expected to occur.

Section 3 gives a description of how the high energy vacua in this landscape are expected to be populated in volume weighted inflation. We discuss here how initial conditions are quickly forgotten, once the highest energy, most rapidly expanding vacua are realised, and subsequently come to dominate the evolution of the universe. We then argue that above a certain high energy boundary the distribution of probability is approximately constant, and isotropically distributed.

In section 4 we consider tunneling events down from this high-energy barrier to a surface one jump up from the anthropic shell. We find that the primary contribution to probability on this low energy shell is due to chains of transitions occurring in a straight line, in one dimension of the flux space. Approximating the discrete space as continuous, we then find an explicit expression for the total tunneling rate down from high energies to the penultimate sphere, before the anthropic one. We find that there exist peaks of probability on this penultimate sphere, and find their position at various different levels of approximation.

Section 5 gives a description of the final transition down into the anthropic shell. This transition is treated separately as the effects of gravity are negligible for this final jump. We find that this last step also produces peaking, but that this peaking is negligible in comparison to that found in section 4 .

A description of the extent of peaking, due to suppressed transitions, can be found in section 6. For a typical set of parameters we find that $99.9999 \%$ of the vacua in the anthropic shell should be expected to lie within a fraction $\sim 10^{-23}$ of its surface area. The selected vacua have one flux much larger than the others. There are ample vacua in the selected set to allow for an anthropic solution of the cosmological constant problem. We also consider in this section the effect of the anthropic life-time bound, already discussed in section 2. The peak of probability is found to be centered on vacua with insufficiently long life-times for observers to appear. If we consider only vacua with life-times $>10$ billion years, then the peaking in probability exerts a pressure favoring the vacua with shorter lives. We find that a typical vacuum, in which observers take 10 billion years to appear, will be most likely to decay approximately 2.2 billion years later.

Section 7 gives a discussion of the results obtained, and how they may be altered by effects that were not included in our model. For example, if multiple branes are allowed to be nucleated simultaneously then the peaking we have found should be expected to be reduced, and to vanish in the limit of indefinitely many branes nucleating simultaneously. We also consider how the required stage of slow-roll inflation, before re-heating, could modify our results.

\section{The Model}

We consider here volume-weighted measures of inflation in the Bousso-Polchinski landscape. We will begin with a brief explanation of these measures in eternal inflation, and then describe 


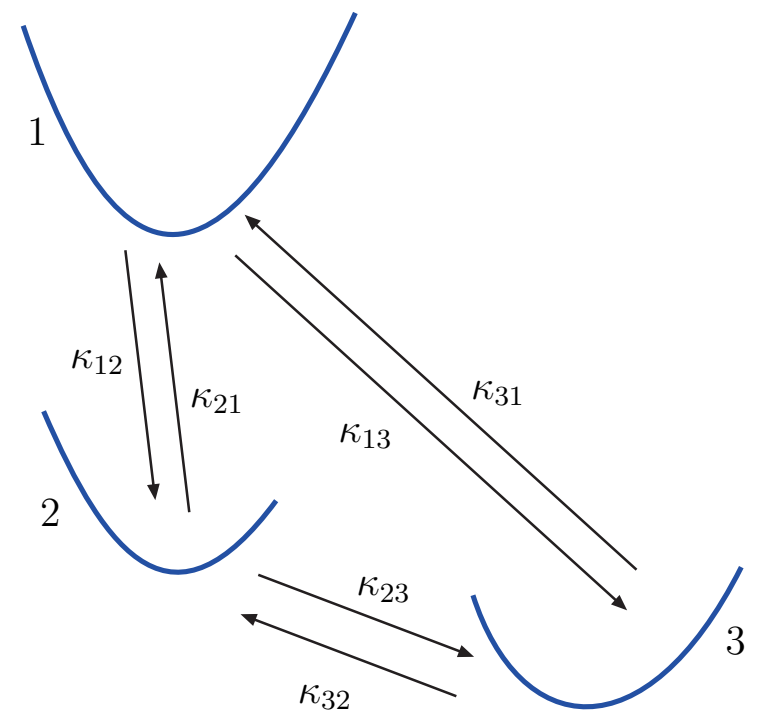

Figure 1: A toy landscape, with three dS vacua. $\kappa_{i j}$ denotes the tunneling rate from vacuum $i$ to vacuum $j$.

the features of the Bousso-Polchinski landscape that are of relevance for our study.

\subsection{Volume Weighting}

The essential feature of volume weighted measures is that they reward those regions which expand most rapidly. That is, in a given increment of time, $\Delta t$, the 3 -volume of each type of vacuum is increased as a function of its rate of expansion, in some proportionate way. Clearly the way in which time is measured is of primary importance here. For definiteness we will define 'time' as being the proper time measured by a congruence of comoving observers. This can be measured in an invariant way in units of the 11-dimensional Planck time, or by equipping each observer with a suitably constructed light clock. Inflation occurs in each vacuum until a tunneling event creates a bubble of new vacuum in the old (or on rare occasions until Minkowski space is reached, if this exists in the theory). The number of points in any particular vacuum that do not eventually undergo a transition to a bubble of new vacuum are a set of measure zero. However, due to the rapid expansion of high-energy vacua, such vanishingly small sets can still come to dominate the evolution of the universe. To make these ideas concrete, we will illustrate this behavior with an example.

Consider a simple landscape of 3 de Sitter vacua, as shown in Figure 1, Here $\kappa_{i j}$ denotes the transition rate from vacuum $i$ to vacuum $j$, and the $H_{i}$ are Hubble constants. We assume that vacuum 1 has a much higher cosmological constant than vacua 2 and 3 , so that $H_{1} \gg H_{2,3}$ and $\kappa_{1 i} \gg \kappa_{i 1}, \kappa_{23}, \kappa_{32}$. If $P_{i}$ is the (unnormalized) probability of finding a given point in the 
vacuum state $i$, then we can describe this dynamical system through a set of coupled ODEs [10], [11:

$$
\begin{aligned}
& \dot{P}_{1}=\kappa_{21} P_{2}+\kappa_{31} P_{3}-\left(\kappa_{12}+\kappa_{13}\right) P_{1}+3 H_{1} P_{1}, \\
& \dot{P}_{2}=\kappa_{12} P_{1}+\kappa_{32} P_{3}-\left(\kappa_{23}+\kappa_{21}\right) P_{2}+3 H_{2} P_{2}, \\
& \dot{P}_{2}=\kappa_{13} P_{1}+\kappa_{23} P_{2}-\left(\kappa_{32}+\kappa_{31}\right) P_{3}+3 H_{3} P_{3} .
\end{aligned}
$$

As long as $H_{1} \gg \kappa_{i j}$ for all $i, j$ (i.e. the highest energy vacuum expands much faster than it decays), the first of these equations becomes

$$
\dot{P}_{1} \simeq 3 H_{1} P_{1} \quad \Rightarrow \quad P_{1} \propto e^{-3 H_{1} t}
$$

The terms dominating the RHS of the second two equations are then those which are proportional to $P_{1}$, as $t \rightarrow \infty$. These equations are solved by

$$
\frac{P_{i}}{P_{1}} \simeq \frac{\kappa_{1 i}}{3 H_{1}} \ll 1 \quad \text { or } \quad \frac{P_{i}}{P_{j}} \simeq \frac{\kappa_{1 i}}{\kappa_{1 j}} .
$$

This confirms that the probability of finding a point in any particular vacuum is proportional to its rate of decay from the highest energy (fastest-growing) vacuum. Essentially, the most rapidly expanding vacuum sources all of the others - it is easier to make the lower vacua by expanding in vacuum 1 and then decaying down, than it is to do so by simply expanding with the smaller Hubble constant of the lower vacua.

These results readily generalize to more complicated systems. First, consider a landscape in which the highest energy vacuum can decay to all other vacua. In this case we have

$$
\frac{P_{i}}{P_{h}} \simeq \frac{\kappa_{h i}}{3 H_{h}} \ll 1 \quad \text { or } \quad \frac{P_{i}}{P_{j}} \simeq \frac{\kappa_{h i}}{\kappa_{h j}},
$$

where $h$ labels the highest energy vacuum. Now consider a landscape in which there exist vacua that cannot be created from direct decays from the highest energy state. In this case the probability of finding a point in the vacuum state $j$ is

$$
\frac{P_{j}}{P_{h}}=\sum_{\substack{\text { all } \\ \text { paths }}} \frac{\kappa_{h a}}{3 H_{h}} \times \frac{\kappa_{a b}}{3 H_{h}} \times \frac{\kappa_{b c}}{3 H_{h}} \times \ldots \times \frac{\kappa_{i j}}{3 H_{h}} \ll 1 .
$$

The chain of $\kappa_{i j} / 3 H_{h}$ gives the probability of starting in the state $h$ and finishing in $j$, following some series of decays through the vacua $a, b, c \ldots$. To obtain the total probability of finding a point in the vacuum $j$ it is then necessary to sum over all possible paths. Clearly some paths will provide more of a contribution than others to this sum. In fact, as long as $\kappa_{i j} \ll H_{h}$, only the shortest decay chains will give a significant contribution. Every chain with additional, unnecessary, intermediary vacua will be suppressed by factors of $\kappa_{i j} / 3 H_{h} \ll 1$. However, if $\kappa_{i j} \sim 3 H_{h}$ then the products of decay are no longer suppressed. In this case the progeny of decaying vacua will be as equally abundant as the vacua from which they decayed. Rapid transitions of this type will not contribute suppressing factors to the decay chains mentioned above. 


\subsection{The Bousso-Polchinski Landscape and Transitions}

We will consider here the Bousso-Polchinski (BP) landscape of M-Theory [2]. This model has the advantages of being relatively simple and, most importantly for our purposes, having known expressions for the transition rates.

The BP landscape consists of a discrete set of vacua, each with a different effective cosmological constant given by

$$
\Lambda=-\Lambda_{0}+\frac{1}{2} \sum_{i=1}^{J} q_{i}^{2} n_{i}^{2},
$$

where $J$ is the number of fluxes, $q_{i}$ the charge associated with each of them and $n_{i}$ the number of units of a particular flux. The discreteness of the $n_{i}$ arises from the quantization of four-form fluxes in M-theory compactifications, and their multiplicity from the geometry of the M-theory compactification - each non-trivial three-cycle giving rise to a different four-form flux. The number of such cycles is generically quite large. This multiplicity allows a much finer spacing of $\Lambda$ than that given by a single flux alone, which typically has a charge only a few orders of magnitude below the Planck scale 2 .

From equation (3) it is clear that for a given choice of $\Lambda$, the fluxes are constrained to lie on a sphere in flux space, of radius $\sqrt{2\left(\Lambda+\Lambda_{0}\right)}$. Taking $0 \leqslant \Lambda \lesssim 10^{-120}$ (in 4D Planck units) we obtain a thin shell of radius $\sim \sqrt{2 \Lambda_{0}}$, which corresponds to the set of vacua in the BP landscape which have an anthropically acceptable cosmological constant (see below).

Transitions in this landscape occur via the nucleation of branes. The transition rates, including gravitational effects, are given by [12]

$$
\kappa_{i j}=A_{i j} e^{-B_{i j}} .
$$

The $A_{i j}$ can be approximated by $A_{i j} \sim 1$ (any effect they have will be exponentially unimportant). Now let us consider the nucleation of bubbles which change the flux through some particular cycle by one unit, from $n_{i}$ to $n_{i}-1$, such that

$$
\left|\Delta \Lambda_{i}\right|=\left(n_{i}-1 / 2\right) q_{i}^{2}
$$

The tunneling rate is then given as the product of its value in flat space, times some gravitational correction, as [13]

$$
B_{i \downarrow}=B_{i \downarrow}^{\text {flatspace }} r(x, y),
$$

where the gravitational correction factor is

$$
r(x, y)=\frac{2\left[(1+x y)-\left(1+2 x y+x^{2}\right)^{1 / 2}\right]}{x^{2}\left(y^{2}-1\right)\left(1+2 x y+x^{2}\right)^{1 / 2}}
$$

and the flat space rate is [14]

$$
B_{i \downarrow}^{\text {flat-space }}=\frac{27 \pi^{2}}{8} \frac{1}{\left(n_{i}-1 / 2\right)^{3} q_{i}^{2}} .
$$


The dimensionless parameters in $r(x, y)$ are given by

$$
x \equiv \frac{3 q_{i}^{2}}{8\left|\Delta \Lambda_{i}\right|}=\frac{3}{8\left(n_{i}-1 / 2\right)} \quad \text { and } \quad y \equiv \frac{2 \Lambda}{\left|\Delta \Lambda_{i}\right|}-1
$$

For our purposes $n_{i}$ will always be $\gg 1$, so that $x \simeq 3 / 8 n \ll 1$. The variable $y$ has a clear interpretation as the energy-level of the parent vacuum. At high energies $\Lambda_{i} \gg\left|\Delta \Lambda_{i}\right|$, so that $y \gg 1$. Conversely, at the lowest energies $\Lambda_{i} \sim\left|\Delta \Lambda_{i}\right|$, so that $y \sim 1$. The combination $x y$ can be interpreted as a measure of distance from the axes of the flux space. Consider the surface defined by $x y=3 / 8$, we then have

$$
x y \simeq \frac{3}{8 n_{i}} \frac{2 \Lambda_{i}}{\left|\Delta \Lambda_{i j}\right|} \simeq \frac{3}{4} \frac{\Lambda}{n_{i}^{2} q_{i}^{2}} \simeq \frac{3}{8} \quad \Rightarrow \quad \sum_{k \neq i} n_{k}^{2} q_{k}^{2} \simeq 2 \Lambda_{0} .
$$

The LHS of this second equation is the perpendicular distance (squared) away from the axis corresponding to the direction in which the transition is occurring. The RHS is the radius (squared) of the anthropic shell. Therefore when $x y \lesssim 3 / 8$ the transition is occurring in a cylinder around this axis, of the same cross-sectional area as the anthropic shell. When $x y \gg 3 / 8$ the transition is occurring well away from the axis.

Useful estimates of tunneling rates can be found in a number of different regimes [7]. Firstly, at high energies far from the axis, when $y \gg 1$ and $x y \gg 1$, it can be seen that

$$
r(x, y) \simeq \sqrt{2}(x y)^{-3 / 2} \ll 1 .
$$

The tunneling rate (5) is then given by

$$
\kappa_{i j} \simeq \operatorname{Exp}\left[-\frac{3 \sqrt{6} \pi^{2} q_{i}}{\Lambda_{i}^{3 / 2}}\right]
$$

This expression is independent of $n_{i}$. Alternatively, closer to the axis, when $y \gg 1$ and $x y \ll 1$, we obtain the Padé approximant

$$
r(x, y) \simeq \frac{1-x y}{1+x y}
$$

This approaches the flat space limit as $x y \rightarrow 0$ :

$$
\kappa_{i j} \sim \operatorname{Exp}\left[-\frac{27 \pi^{2}}{8} \frac{1}{n_{i}^{3} q_{i}^{2}}\right] .
$$

Another regime of interest is at low energy, when $y \sim 1$ : that of the final transition, down into the anthropic shell. In this limit [12]

$$
r(x, y) \simeq\left(1+x^{2}\right)^{-2}=\frac{\left(n_{i}-1 / 2\right)^{2}}{\left(n_{i}-1 / 8\right)^{2}} \simeq 1
$$




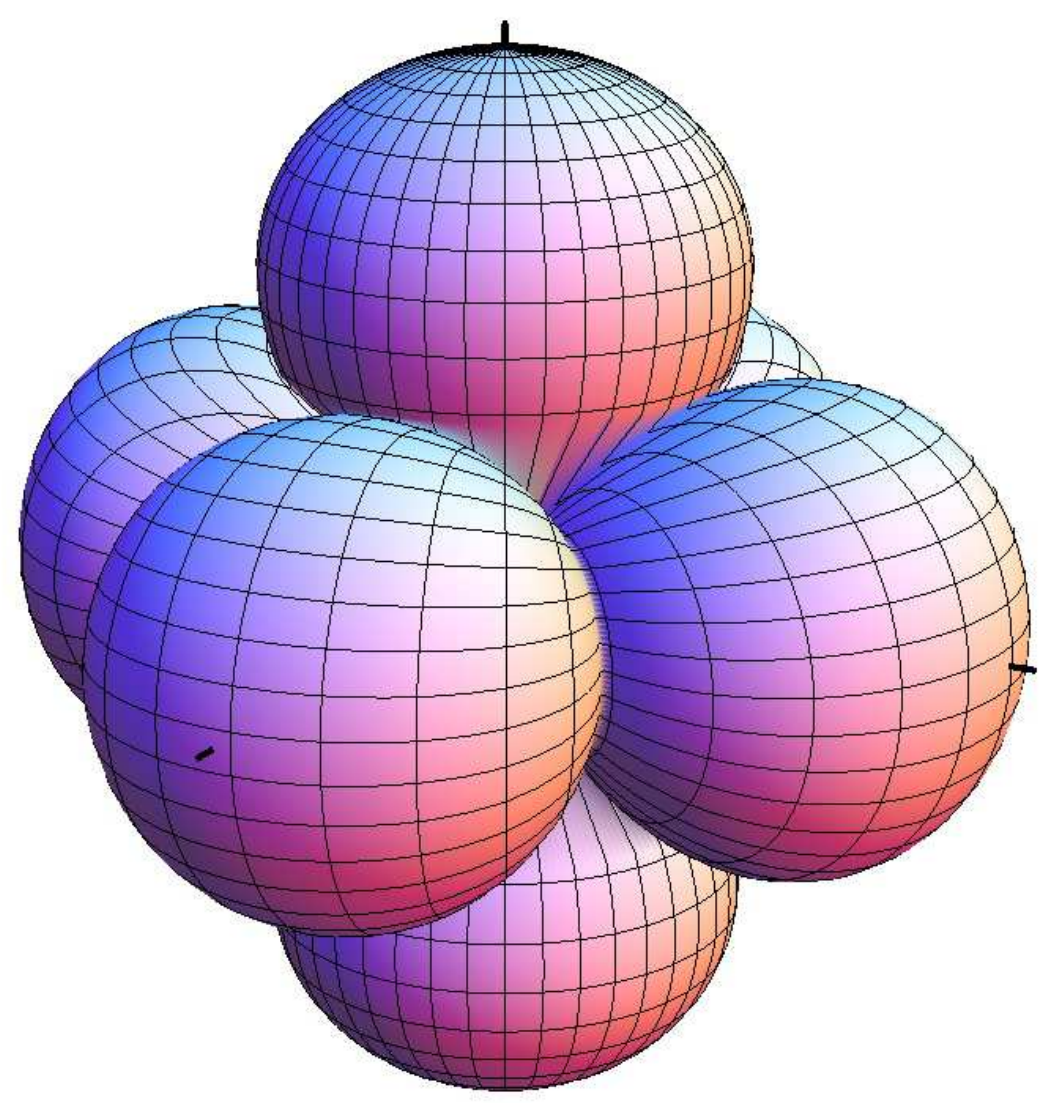

Figure 2: A plot of the flat space tunneling rate (12), as a function of angular coordinates in three dimensions. The tunneling rate is represented here as being proportional to the distance from the origin.

such that the gravitational correction is vanishingly small. As long as this condition is met, we can then approximate the tunneling rate down into the anthropic shell by equation (12).

The flat space tunneling rate is shown graphically, as a function of angular coordinates, in figure 2. It can be seen that $\kappa_{i j}$ is significantly larger near the axes of this space, even in these few dimensions. In more dimensions, the contrast between maximum and minimum is increased. This expression for $\kappa_{i j}$ will be used below to show that even a single transition (the last one) can produce considerable clustering of probability around the axes of the flux space. As the radius of the sphere being considered (and hence the energy level) is increased, gravitational corrections must increasingly be taken into consideration. The effect of gravity is to reduce the contrast between maxima and minima, smoothing the distribution of $\kappa_{i j}$ and producing a plot that looks more like a sphere.

As well as nucleating branes of single charge, one may also wish to consider the possibility of nucleating stacks of branes simultaneously. A study of such events has been carried out by 
Garriga and Megevand [15]. In this work it is concluded that the instantons for nucleating stacks of branes may well not exist, but if they do then the tunneling rates for such events will be approximately given by $B_{i j}^{(k)} \simeq k B_{i j}$, where $k$ is the number of branes in the stack and $B^{(k)}$ is the exponent of the tunneling rate for nucleating the stack. This formula was found in the limit that the bubble wall is much less than the Hubble rate of the parent vacuum, in which case the thin-wall approximation can be used. When a sufficiently large stack is nucleated (again, if this is possible), then the thin wall approximation is violated and the bound on the minimum possible tunneling rate [16] is saturated. In this limit, the exponent of the tunneling rate was found to be $B_{i j}^{(k)} \simeq \frac{8 \pi^{2}}{\Lambda_{i}}$. This rate is independent of the everything except the energy of the parent vacuum, and so once it is met all unlikely tunneling events proceed with this minimum probability, if instantons for these transitions exist at all.

The possibility of nucleating stacks of branes significantly alters the dynamics of inflation, if it is allowed to occur. We consider it beyond the scope of the present study to perform a detailed analysis of this phenomenon here. Instead, we will first proceed using the standard lore of allowing only transitions between nearest neighbors in the BP landscape. After analyzing this situation we will then reflect on how nucleating multiple branes simultaneously will change this picture. We leave it to future studies to determine how likely these events really are.

\subsection{Anthropic Considerations}

In volume weighted measures of inflation the majority of the 3-volume of the universe, and the majority of new bubbles formed, are at very high energies. However, no observers can exist in these vacua. Firstly, the famous anthropic bound on the cosmological constant tells us that anthropically acceptable vacua must lie within the range $0 \lesssim \Lambda \lesssim 10^{-120}$ in order for structure to be able to form [17] (we neglect the possibility of small negative cosmological constant [18], for simplicity). Secondly, these high energy vacua decay far too quickly for structure to form. If we are interested in the likely locations in the landscape for observers to find themselves we should therefore not ask simply what are the most likely vacua, but instead what are the most likely vacua that (a) have a suitably low cosmological constant, and (b) are suitably long-lived 5 .

\subsubsection{Cosmological constant bound}

As we mentioned in the preceding subsection, the condition (a) is tantamount to considering only vacua within a thin shell of width $10^{-120}$, and radius $\sqrt{2 \Lambda_{0}}$. Any points further from the origin than this have $\Lambda>10^{-120}$, and so do not allow for the formation of structure. Any points

\footnotetext{
${ }^{5}$ There are a number of other anthropic bounds that should also be applied from, for example, the fine structure constant and Newton's constant. For simplicity, and due to the limited nature of our model, we will not consider all of these bounds here.
} 
closer to the origin are collapsing AdS vacua. The volume, $V$, of this shell is then given by

$$
V=\frac{2 \pi^{J / 2}}{\left(\frac{J}{2}-1\right) ! J}\left[\left(\sqrt{2} \sqrt{\Lambda_{0}+10^{-120}}\right)^{J}-\left(\sqrt{2} \sqrt{\Lambda_{0}}\right)^{J}\right] \simeq \frac{10^{-120}(2 \pi)^{J / 2} \Lambda_{0}^{J / 2}}{\left(\frac{J}{2}\right) !}
$$

The density of points in the flux space is $\left(\prod_{i}^{J} q_{i}\right)^{-1}$, so that the condition for more than one point in this anthropic shell is

$$
\frac{10^{-120}(2 \pi)^{J / 2} \Lambda_{0}^{J / 2}}{\left(\frac{J}{2}\right) ! \prod_{i}^{J} q_{i}}>1
$$

\subsubsection{Life-time bound}

The condition (b) requires that the time scale $\tau_{i j}=\kappa_{i j}^{-1}$ is less than the time required for structure to form, and life to evolve. This is clearly a very difficult time to specify precisely. For definiteness we will require the bound that the vacuum should have a lifetime $\gtrsim 10 G y r$, corresponding to

$$
\kappa_{i j} \lesssim e^{-138}
$$

The location of this boundary could be shifted, depending on the time at which observers are deemed to appear in any particular theory. Transitions out of any point that satisfies condition (a) will be adequately described by the flat space rate, (12). This rate is at a minimum along the 'diagonal', where $J n_{D}^{2} q_{i}^{2}=2 \Lambda_{0}$. The condition for suitable stability of these points is given by

$$
q_{i} J^{3 / 2} \gtrsim 13 \Lambda_{0}^{3 / 2}
$$

This condition on the slowest transitions rates is the requirement that there exist any points that live longer than 10Gyr. The condition that all points in the anthropic shell are this stable requires a condition on the fastest transition rate, at the poles. Here $n_{P}^{2} q_{i}^{2}=2 \Lambda_{0}$, and the tunneling rate (12) gives

$$
q_{i} \gtrsim 13 \Lambda_{0}^{3 / 2}
$$

The anthropic conditions (a) and (b) therefore give us acceptable ranges of the parameters $q_{i}$ and $\Lambda_{0}$, in terms of the number of dimensions $J$. Strictly, these relations should not be considered independent of each other as we require not only a minimum of one point in the anthropic shell and one region in this shell where points are stable, but one stable point in the shell. The combination of conditions (13) and (14) can then be considered a necessary condition, and the combination of conditions (13) and (15) a sufficient one. These conditions are summarized in the table below, where it is assumed that all charges are approximately equal (so that all $q_{i} \simeq q$ ).

It can be immediately seen, for reasonable parameters, that the condition for all points in the anthropic shell to be stable is often not satisfied. This means that the regions near the poles of this shell are not long-lived enough for observers to evolve. It can also be seen that for 


\begin{tabular}{llll}
\hline & $\begin{array}{l}\text { some points } \\
\text { stable }\end{array}$ & $\begin{array}{l}\text { all points } \\
\text { stable }\end{array}$ & $\begin{array}{l}\text { enough points } \\
\text { in shell }\end{array}$ \\
\hline $\mathrm{J}$ & $q \Lambda_{0}^{-3 / 2} \gtrsim$ & $q \Lambda_{0}^{-3 / 2} \gtrsim$ & $q^{-1} \Lambda_{0}^{1 / 2} \gtrsim$ \\
\hline 100 & 0.013 & 13 & 28 \\
250 & 0.0033 & 13 & 8.3 \\
500 & 0.0012 & 13 & 6.7 \\
1000 & 0.00041 & 13 & 7.2 \\
2000 & 0.00015 & 13 & 8.8 \\
\hline
\end{tabular}

fixed $\lambda_{0}$ (13) provides an upper bound on $q$, and (14) provides a lower bound. Similarly, for fixed $q$ (13) provides a lower bound on $\Lambda_{0}$, whilst (14) provides an upper bound.

We expect the bare cosmological constant to be of order unity, and the charges $q$ to be a couple of orders of magnitude below this scale. Reasonable values for the dimensionality of the phase space are 100 to 1000. For definiteness in what follows, we will often consider a model in which $q=10^{-2}, \Lambda_{0}=1 / 2$ and $J=500$. This satisfies the conditions (13) and (14), but not (15). The condition (15) could be satisfied by considering a smaller $\Lambda_{0}$.

\section{Populating at High Energies}

One of the benefits of volume weighted measures of inflation is that there exists a stable latetime distribution, which can be reached from a broad range of initial conditions: Once this 'stationary' regime is achieved, the initial conditions of the system are quickly forgotten. All that is required for the universe to achieve stationarity is that at least one part of it should reach the highest energy states. Although this may require unlikely upwards tunneling events, the rapid expansion of the new higher-energy vacua produced can often be more than enough to offset the improbability of such events occurring.

Of course there exists an upper boundary beyond which the semi-classical methods we employ cannot be usefully applied. This is the Planck boundary, beyond which a full quantum theory of gravity is required for an adequate description of the space-time (if this concept survives). We will not consider such regimes here, but will instead restrict ourselves to considering only sub-Planckian regions. We will now argue that on high energy shells, of constant $\Lambda$, probability will be distributed approximately isotropically.

In a landscape with many high energy vacua the evolution of the universe, according to volume weighted measures, will not be dominated by a single high-energy vacuum, as may initially be suspected, but rather by the combined expansion of the many highest energy vacua 6 .

\footnotetext{
${ }^{6}$ We thank Andrei Linde for making this point clear to us.
} 
The effect of the expansion of these vacua will give an exponentially growing probability

$$
P_{h} \sim e^{3 H_{h} t},
$$

which will be shared by the lower energy vacua in the manner outlined in section 2 . Here $H_{h}$ is the effective Hubble rate of the highest energy vacua, which we expect to be near Planckian. We may now ask ourselves what the condition is for a particular vacuum to find itself in some 'smeared out' region at high energy, where all $P \simeq P_{h}$. To answer this consider the evolution equation

$$
\begin{aligned}
\dot{P}_{i} & =\sum_{j} \kappa_{j i} P_{j}-\sum_{k} \kappa_{i k} P_{i}+3 H_{i} P_{i} \\
\simeq 3 H_{h} P_{i} & \simeq \sum_{j} \kappa_{j i} P_{h}-\sum_{k} \kappa_{i k} P_{i}+3 H_{i} P_{i} .
\end{aligned}
$$

In going from the first to second line we have assumed that all neighboring vacua to $P_{i}$ have approximately $P_{j} \simeq P_{h}$, i.e. that our chosen vacuum is in the smeared out region. This assumption will be consistent only if $P_{i} \simeq P_{h}$, in which case the vacuum can consistently be described as being in a 'smeared out' region at high-energy. To see if this is true we can rearrange the equation above to obtain

$$
\frac{P_{h}}{P_{i}} \simeq \frac{3 \Delta H+\sum \kappa_{i \downarrow}}{\sum \kappa_{\downarrow i}},
$$

where $\Delta H \equiv H_{h}-H_{i}$. Now, if $3 \Delta H \lesssim \sum \kappa_{i \downarrow}$, then

$$
\frac{P_{h}}{P_{i}} \simeq \frac{\sum \kappa_{i \downarrow}}{\sum \kappa_{\downarrow i}} \sim O(1) .
$$

In this case we have $P_{i} \sim P_{h}$, and the vacuum under consideration is in the smeared out region. (The order of magnitude here is due to the small difference in energy levels between neighboring high-energy vacua in the BP landscape). Alternatively, when $3 \Delta H \gg \sum \kappa_{i \downarrow}$, we have

$$
\frac{P_{h}}{P_{i}} \simeq \frac{3 \Delta H}{\sum \kappa_{\downarrow i}} \gg 1,
$$

and $P_{i} \ll P_{h}$. In this case the vacuum we are considering cannot consistently be assumed to be in the smeared out region.

Furthermore, transitions between the highest energy vacua in the BP landscape will be only very weakly suppressed, with $\kappa_{i j} \sim O(1)$. As such, the value of $\kappa_{i j} / 3 H_{h}$ will also be $O(1)$ and the ratio of probabilities given by (2) will be

$$
\frac{P_{j}}{P_{h}} \sim O(1) .
$$


The products of any such decay will then be (approximately) as equally abundant as the vacua from which they decayed. If the highest energy vacua decay at these rates then the whole of the high-energy part of the landscape will very quickly become populated with an approximately flat distribution of probability.

We have identified here reason for thinking that the highest energy levels of the BP landscape should have a probability distribution that is 'smeared out' (i.e. isotropically distributed). This smearing process erases any anisotropy, or favoring of any particular point or energy level in the initial conditions of the universe - as long as the highest energy vacua can be reached, we are left with a landscape that is populated at high energies by an approximately flat distribution of probability. Clearly this is a very different regime to the low energy one, in which tunneling events are suppressed by large factors of $3 H_{h} / \kappa_{i j} \gg 1$ : Hence, there exists a boundary above which we can expect tunneling rapid enough to produce a flat probability distribution, and below which tunneling is heavily suppressed.

\section{Tunneling from High to Low Energies}

We will now consider how tunneling from high energies down to lower energies will proceed. First it is necessary to determine which paths will contribute the highest probability flux onto the anthropic shell. The shortest path from any point on the anthropic shell to the 'smeared out' surface, described above, will be a straight line extending in one dimension of the flux space. Any deviations from this shortest path will add extra factors of $\kappa_{i j} / 3 H_{h}$, which will suppress their relative contribution to the overall flux arriving at our point. It is now necessary to determine whether this suppression will be sufficient to beat the combined contribution of the potentially large number of paths which are slightly longer, and less favorable.

The degree to which a longer path can be considered less favorable depends upon where it deviates from the straight line, shortest path, and whereabouts in the flux space the shortest path is located. For example, a straight-line shortest path which is parallel, and close to an axis of the flux space will have very little contribution from any other (longer) paths. This is due to the vacua along such a trajectory having one large flux, and the rest very small. Transitions in directions with small fluxes associated with them will then only progress at very low rates, compared to those in the direction of the single large flux. The shortest path, which is oriented in the direction of the large flux, will then consist of a series of rapid transitions. Any longer path will necessarily include additional transitions in a direction associated with a smaller flux, and so will be heavily suppressed. Away from the axes extra transitions at or near the surface beyond which rates are no longer suppressed will not incur a large penalty. We will treat this high energy surface as an initial boundary from which tunneling down to the anthropic shell begins. Any unsuppressed transitions before this surface can then be considered as contributing to the boundary, but not favoring any particular path thereafter. We will show that our results are not heavily dependant on the exact location of such a boundary. Extra deviations away from the shortest path at low energies will incur a large penalty, which will be enough to make 


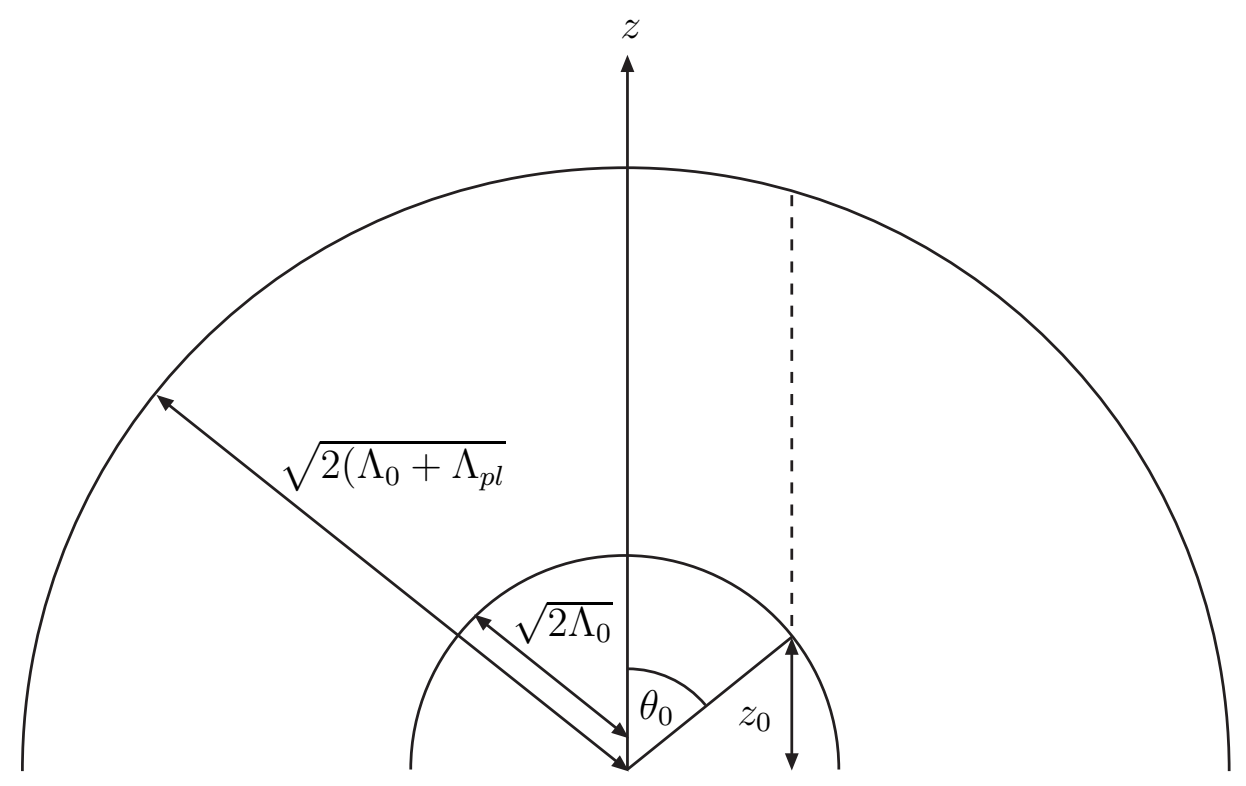

Figure 3: A schematic of the shortest path from high energies to the penultimate sphere, parallel to the $z$ axis.

any such contribution to the total probability negligible. We therefore expect the shortest path from the high-energy boundary to be a good approximation to the probability of arriving at an acceptable point in the anthropic shell, both near and far from the axes of the phase space.

We now need the appropriate transition rates along these shortest paths. Although the approximation to the gravitational correction (9) is the appropriate one for most of the highenergy transitions (in a high dimensional landscape), it is not the one in which we are interested here. A straight-line extending on one dimension of the flux space, from the anthropic shell to high energies, will always be contained within a cylinder about one of the axes, of cross-sectional area less than that of the anthropic shell itself. The appropriate gravitational correction is therefore given by (11) along such a path, until the last transition where it is given by (12). In this section we will consider the regime where (11) is valid. The effect of the last transition will be considered separately in the next section.

The tunneling rate prescribed by (11) is

$$
\kappa_{i j} \simeq \operatorname{Exp}\left[-\frac{27 \pi^{2}}{8 n_{i}^{3} q_{i}^{2}}\left\{\frac{4 n_{i}^{2} q_{i}^{2}-3 \Lambda_{i}}{4 n_{i}^{2} q_{i}^{2}+3 \Lambda_{i}}\right\}\right]
$$

and the total probability of tunneling down from high energies to the penultimate sphere, before the anthropic one, is then given by the product of these tunneling rates along the shortest path, shown schematically in figure 3 .

The value of $\Lambda_{i}$ along this path is

$$
\Lambda_{i}=\frac{1}{2} n_{i}^{2} q_{i}^{2}-\frac{1}{2} z_{0}^{2}
$$


where $z_{0}^{2} \equiv 2 \Lambda_{0} \cos ^{2} \theta_{0}$ and $\theta_{0}$ is shown in figure 3 . The value of $n_{i} q_{i}$, for any $i$, is

$$
n_{i} q_{i}=q_{i}\left(n_{0 i}+j\right)=z_{0}+j q_{i}
$$

where $n_{0 i}$ is the value of $n_{i}$ in the anthropic shell, and $j$ is the number of flux quanta in $z$ away from that point. Now the total supression along this path is given by

$$
\kappa_{T} \equiv \prod_{i}^{J} \kappa_{i \downarrow}=\operatorname{Exp}\left[-\frac{27 \pi^{2} q_{i}}{8} \sum_{j=2}^{N}\left(\frac{4 n_{i}^{2} q_{i}^{2}-3 \Lambda_{i}}{4 n_{i}^{2} q_{i}^{2}+3 \Lambda_{i}}\right) \frac{1}{n_{i}^{3} q_{i}^{3}}\right]
$$

where $\Lambda_{i}$ and $n_{i} q_{i}$ are given by (16) and (17), and $N$ is the number of transitions involved in tunneling from high energy to low energy. In a continuum approximation this expression can be approximated by

$$
\begin{aligned}
\kappa_{T} & \simeq \operatorname{Exp}\left[-\frac{27 \pi^{2} q_{i}}{8} \int_{3 / 2}^{N+1 / 2}\left(\frac{4 n_{i}^{2} q_{i}^{2}-3 \Lambda_{i}}{4 n_{i}^{2} q_{i}^{2}+3 \Lambda_{i}}\right) \frac{d j}{n_{i}^{3} q_{i}^{3}}\right] \\
& \simeq \operatorname{Exp}\left[-\frac{27 \pi^{2}}{8}\left[\frac{8 \ln \left\{\frac{6 \Lambda_{i}}{n_{i}^{2} q_{i}^{2}}+8\right\}}{3\left(n_{i}^{2} q_{i}^{2}-2 \Lambda_{i}\right)}+\frac{1}{2 n_{i}^{2} q_{i}^{2}}\right]_{3 / 2}^{N+1 / 2}\right] .
\end{aligned}
$$

As we have already discussed, the leading contribution to this quantity will be the low energy limit, where extra transitions are heavily suppressed. The contribution from extra transitions at high energy will be negligible, and correspondingly we take $N \rightarrow \infty$. In this limit we then have

$$
\kappa_{T} \simeq \operatorname{Exp}\left[\frac{27 \pi^{2}}{8}\left(\frac{8}{3 z_{0}^{2}} \ln \left(1-\frac{12 z_{0}^{2}}{11\left(2 z_{0}+3 q_{i}\right)^{2}}\right)+\frac{2}{\left(2 z_{0}+3 q_{i}\right)^{2}}\right)\right] .
$$

The limit $N \rightarrow \infty$ is further justified in appendix $\mathrm{A}$, where integrations are performed to finite values of $N$. It is found that the quantities we are interested in are largely insensitive to the exact location of the high-energy boundary, and so we are justified in approximating it to be at infinity. For $z_{0} \gg q_{i}$ the expression above becomes

$$
\kappa_{T} \simeq \operatorname{Exp}\left[-\frac{27 \pi^{2}}{8 z_{0}^{2}}\left(\frac{8}{3} \ln \left(\frac{11}{8}\right)-\frac{1}{2}\right)\right] \simeq \operatorname{Exp}\left[-\frac{12}{z_{0}^{2}}\right] .
$$

It can be clearly seen that the tunneling rate is significantly faster to points in the anthropic shell with large $z$ (i.e. close to the axes, away from the diagonal). This is due to the supression associated with the extra transitions required to get to the points further from the axes. We will now use this expression for the tunneling rates to determine where on the sphere a 'typical' vacuum is located. In order to do this we will need to balance the faster transition rates at the poles of the sphere, with the increased volume along the diagonals. 


\subsection{Expectation Values}

In order to consider the distribution of probability on a sphere it is useful to determine the moments

$$
\left\langle f^{2}\right\rangle \equiv \int f^{2} \kappa_{T} d \Omega
$$

where $f=f\left(x_{i}, z\right)$ is any function of $x_{i}$ and $z$ on the sphere, and $d \Omega$ is the surface element of a hypersphere. This expression takes into account not only the effect of the transition rate $\kappa_{T}$, but also the effect of increasing surface area away from the axes.

We can consider the penultimate sphere as being divided into $2 J$ symmetric sectors, each centered around one of the $2 J$ axes of the space. Each of these sectors corresponds to the region $\sigma$ on the surface of the penultimate sphere which is closest to the axis about which it is centered. $z$ is then given, in each respective region, as the corresponding value of the Cartesian coordinate along its central axis, and the whole sphere is built up by evaluating $\left\langle n^{2}\right\rangle$ in any one of the regions and multiplying it by $2 J$. The integral that we are now required to evaluate is

$$
\left\langle f^{2}\right\rangle=\int_{0}^{1} d z \prod_{i}^{J-1} \int_{-z}^{z} d x_{i} f^{2} e^{-12 / z^{2}} \delta\left(\sum_{i}^{J-1} x_{i}^{2}+z^{2}-1\right),
$$

where we have taken $\Lambda_{0}=1 / 2$. The Cartesian coordinates $x_{i}$ correspond to all of those directions which are orthonormal to the central axis $z$. The limits of integration, $-z$ and $z$, then pick out the sector discussed above. Included in the integrand is a delta function intended to select the surface at $\sim \sqrt{2 \Lambda_{0}}=1$, which corresponds to the anthropic shell. Here $\left\langle f^{2}\right\rangle$ is being evaluated in any one of the $2 J$ symmetric regions. We will be interested in the choices $f=z$ and $f=x$, where $x$ is any Cartesian coordinate orthonormal to $z$. The moments $\left\langle z^{2}\right\rangle$ and $\left\langle x^{2}\right\rangle$ then give us the expectation values of these quantities, and their ratio gives us a measure of the peaking of probability on the anthropic sphere due to tunneling down from high energy to the penultimate sphere before the anthropic one.

In appendix $\mathrm{B}$ we find that the expectation values for $\left\langle z^{2}\right\rangle$ and $\left\langle x^{2}\right\rangle$ can be given in terms of one-dimensional integrals as

$$
\left\langle z^{2}\right\rangle=\frac{2}{\Gamma\left(\frac{J+2}{2}\right)} \int_{0}^{\infty} d y y^{2}\left[\frac{\sqrt{\pi} y}{\sqrt{12+y^{2}}} E\left(\sqrt{12+y^{2}}\right)\right]^{J-1} e^{-\left(12+y^{2}\right)}
$$

and

$$
\begin{aligned}
&\left\langle x^{2}\right\rangle=\frac{2}{\Gamma\left(\frac{J+2}{2}\right)} \int_{0}^{\infty} d y y^{2}\left(\frac{1}{2\left(12+y^{2}\right)}-\frac{e^{-\left(12+y^{2}\right)}}{\sqrt{\pi} \sqrt{12+y^{2}} E\left(\sqrt{12+y^{2}}\right)}\right) \\
& \times\left[\frac{\sqrt{\pi} y}{\sqrt{12+y^{2}}} E\left(\sqrt{12+y^{2}}\right)\right]^{J-1} e^{-\left(12+y^{2}\right)} .
\end{aligned}
$$




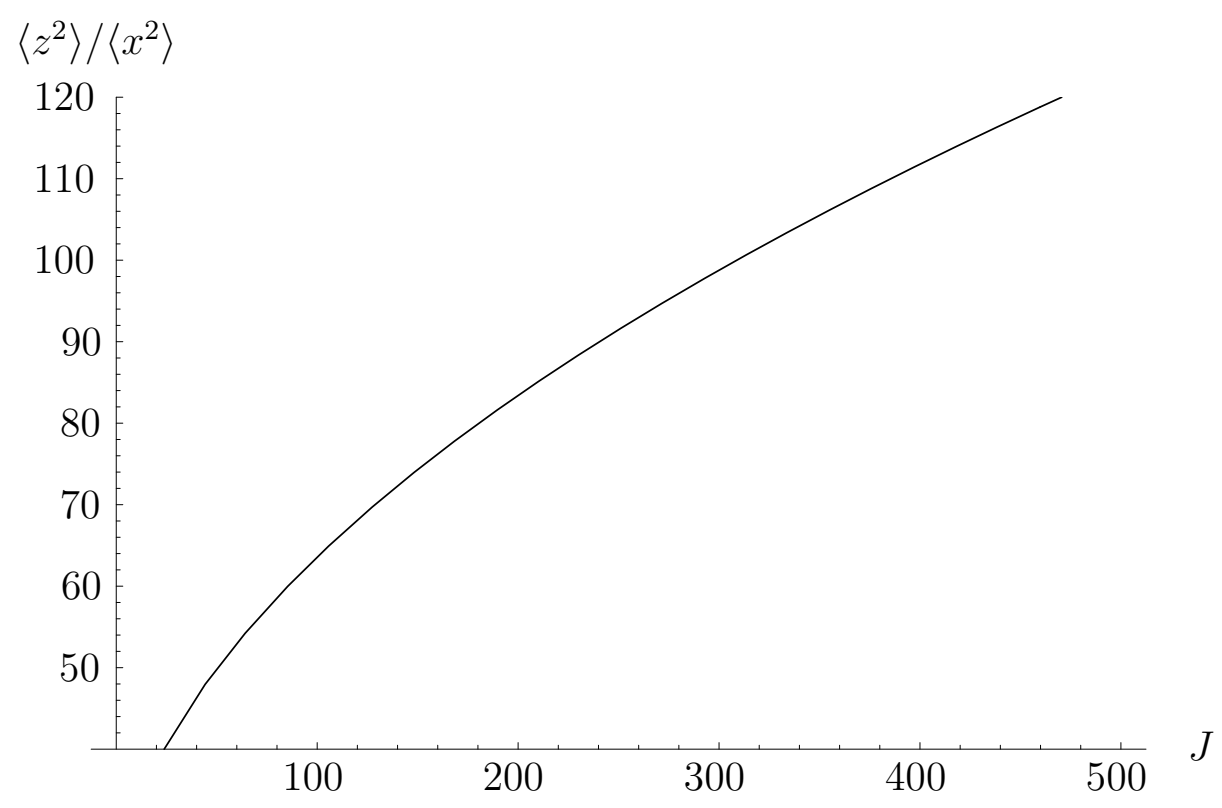

Figure 4: A plot of $\left\langle z^{2}\right\rangle /\left\langle x^{2}\right\rangle$ as a function of the dimensionality of the flux space, $J$.

Here the function $E(p)$ is the integral of a Gaussian distribution, or error function, given by $2 \pi^{-1 / 2} \int_{0}^{p} e^{-q^{2}} d q$.

The measure of peaking that we are interested in is given by the ratio of these two expressions, $\left\langle z^{2}\right\rangle /\left\langle x^{2}\right\rangle$. This is shown graphically in figure 4. It can be seen from this plot that the peaking produced by tunneling down to the penultimate sphere is extremely sharp, and increases with the dimensionality of the flux space. A flat distribution of probability would produce the result $\left\langle z^{2}\right\rangle /\left\langle x^{2}\right\rangle=1$. The large values of this quantity here show that there exists a strong peak in probability on the anthropic shell.

\subsection{The Single Peak Approximation}

We are now in a good position to investigate approximations that will make these quantities more transparent. A particularly useful simplification is to extend the domain of integration of equation (21), to make it independent of $z$. If we were to allow the limits of integration for the coordinates $x_{i}$ to run from -1 to 1 , instead of $-z$ to $z$, then this would allow us to transform to a hyper-cylindrical coordinate system in which $J-1$ of the dimensions could be integrated over in a straightforward fashion. This would leave a simple one-dimensional integral that can be solved by saddle point approximation. We will call this the single peak approximation, as we will be integrating over a larger region that should contain several peaks of probability; we will, however, ignore all but the single central peak of the initial region and show that this is a good approximation to integrating over the correct domain, as in (21). The reason why this is a plausible approximation to consider is that the rate of transitions (19) can be seen to become 
very small at low $z$. The extra region being integrated over in the single peak approximation is all at low $z$, and so it is a reasonable proposition to ask if integrating over such an extended domain yields similar results to integrating over the actual domain of interest. We will argue that it does.

The single peak approximation yields the results

$$
\begin{aligned}
\left\langle\bar{z}^{2}\right\rangle & =\frac{2}{\Gamma\left(\frac{J+2}{2}\right)} \int_{0}^{\infty} d z \prod_{i}^{J-1} \int_{-\infty}^{\infty} d x_{i} z^{2} e^{-\left(\sum_{i} x_{i}^{2}+z^{2}\right)\left(1+12 z^{-2}\right)} \\
& =\frac{2}{\Gamma\left(\frac{J+2}{2}\right)} \int_{0}^{\infty} d z z^{2}\left[\frac{\sqrt{\pi} z}{\sqrt{12+z^{2}}}\right]^{J-1} e^{-\left(12+z^{2}\right)}
\end{aligned}
$$

which can be compared to equation (22), and

$$
\begin{aligned}
\left\langle\bar{x}^{2}\right\rangle & =\frac{2}{\Gamma\left(\frac{J+2}{2}\right)} \int_{0}^{\infty} d z \prod_{i}^{J-1} \int_{-\infty}^{\infty} d x_{i} x^{2} e^{-\left(\sum_{i} x_{i}^{2}+z^{2}\right)\left(1+12 z^{-2}\right)} \\
& =\frac{2}{\Gamma\left(\frac{J+2}{2}\right)} \int_{0}^{\infty} d z \frac{z^{2}}{2\left(12+z^{2}\right)}\left[\frac{\sqrt{\pi} z}{\sqrt{12+z^{2}}} E\left(\sqrt{12+z^{2}}\right)\right]^{J-1} e^{-\left(12+z^{2}\right)},
\end{aligned}
$$

which can be compared to equation (23). We denote quantities evaluated in the single peak approximation by an over-bar. The fractional error of considering this approximation can then be written as

$$
\delta \equiv \frac{\left\langle z^{2}\right\rangle /\left\langle x^{2}\right\rangle-\left\langle\bar{z}^{2}\right\rangle /\left\langle\bar{x}^{2}\right\rangle}{\left\langle z^{2}\right\rangle /\left\langle x^{2}\right\rangle}
$$

This quantity is vanishingly small for all $J$, showing that the single peak approximation is very good.

\subsection{Analytic Approximations}

Using the single peak approximation we will now find analytic expressions for these moments. Recall the tunneling rate is given by

$$
\kappa_{T} \sim \operatorname{Exp}\left[-\frac{12}{z^{2}}\right]=\operatorname{Exp}\left[-\frac{K}{z^{2}}\right]
$$

where $K=12$, and to be concise we have replaced $z_{0}$ with $z$. The normalization of the probability distribution is then

$$
N=\int \kappa d \Omega=2 J \int_{0}^{1} d z \prod_{i}^{J-1} \int_{-1}^{1} d x_{i} e^{-K / z^{2}} \delta\left(\sum_{j}^{J-1} x_{i}^{2}+z^{2}-1\right)
$$


In the single peak approximation, the limits on the $x_{i}$ integrals are replaced with -1 and 1 . Changing coordinates from Cartesian to hyper-cylindrical, and defining $r^{2}=\sum_{i}^{J-1} x_{i}^{2}$, we can rewrite $N$ as

$$
N=2 J V_{J-2} \int_{0}^{1} d z \int_{0}^{1} d r r^{J-2} e^{-K / z^{2}} \delta\left(r^{2}+z^{2}-1\right),
$$

where $V_{J-2}$ is the volume of a $(J-2)$-sphere. Eliminating the delta function with the $r$-integral leaves us with an integral over $z$ :

$$
N=2 J V_{J-2} \int_{0}^{1} d z\left(1-z^{2}\right)^{\frac{J-3}{2}} e^{-K / z^{2}} \sim 2 J V_{J-2} \int_{0}^{1} d z e^{-\left(K / z^{2}-\frac{J}{2} \ln \left(1-z^{2}\right)\right)} .
$$

This integral can be approximated by a saddle point, which is at

$$
z^{2}=\frac{K}{J}\left(-1+\sqrt{1+\frac{2 J}{K}}\right) \sim \sqrt{\frac{2 K}{J}}=\left(\frac{24}{J}\right)^{1 / 4} .
$$

At first glance it may seem that this result is in contradiction with the validity of the single peak approximation. After all, $z$ is small for any reasonable $J(\tilde{C} \lesssim 1)$ and therefore $r$ is close to 1 . However, although $z$ is small, a typical $x_{i}(\sim 1 / \sqrt{J})$ is much smaller and so the peak in the distribution on the sphere is, in general, closer to the $z$-axis than to any of the other $x_{i}$ axes. This issue is discussed in more detail in section 6 .

We can similarly calculate the expectation values of powers of the fluxes:

$$
\left\langle z^{k}\right\rangle=\frac{1}{N} V_{J-2} \int_{0}^{1} d z z^{k}\left(1-z^{2}\right)^{\frac{J-3}{2}} e^{-K / z^{2}} \sim \frac{1}{N} V_{J-2} \int_{0}^{1} d z e^{-\left(K / z^{2}-\frac{J}{2} \ln \left(1-z^{2}\right)-k \ln (z)\right)} .
$$

This should be normalized using the $N$ calculated above. The integrand is again extremized at $z \sim\left(\frac{2 K}{J}\right)^{1 / 4}$ (with an $O\left((2 K / J)^{1 / 4}(k / J)\right)$ correction). Substituting this back into the integral above, and evaluating the saddle point, gives us

$$
\left\langle z^{k}\right\rangle \sim\left(\frac{2 K}{J}\right)^{k / 4}=\left(\frac{24}{J}\right)^{k / 4} .
$$

Here the normalization $N$ cancels the exponential, the remaining integral and various other numerical factors. In a flat measure the same expectation value is given by

$$
\left\langle z^{k}\right\rangle_{\text {flat }}=\frac{V_{J-2}}{N_{\text {flat }}} \int_{0}^{\infty} d r\left(1-r^{2}\right)^{(k-1) / 2} r^{J-2} .
$$

The scaling with $J$ can again be obtained, and is now found to be

$$
\left\langle z^{k}\right\rangle_{\text {flat }} \sim\left(\frac{1}{J}\right)^{k / 2}
$$


Symmetry considerations then give the expected values of the remaining fluxes as

$$
\left\langle x_{i}^{k}\right\rangle \sim \frac{1}{J^{k / 2}}
$$

and a typical flux vector can be estimated as

$$
q \vec{n} \sim\left(\left(\frac{2 K}{J}\right)^{1 / 4}, \frac{1}{J^{1 / 2}}, \ldots, \frac{1}{J^{1 / 2}}\right)
$$

which, for $J=500$ and $q=10^{-2}$, is

$$
\vec{n} \sim(45,4.5, \ldots, 4.5) .
$$

Therefore, the most likely vacua produced by tunneling down from high energies to the penultimate sphere are those with one flux approximately ten times as large as the others. The spread of probability about this most likely point will be addressed in section 6 . Here we have assumed that all charges are the same. Allowing different charges will produce sharper peaking near the axes associated with smaller $q$.

\section{Transitions into the Anthropic Shell}

Having calculated the probability of transitioning down onto the penultimate sphere, above the anthropic one, we will now evaluate the effect of the last jump. The final transition rates into the anthropic shell are well approximated by the flat space rates

$$
\kappa_{F} \sim e^{-\tilde{C} / z^{3}}
$$

where $z$ denotes the value of the Cartesian coordinate in the direction of transition, and $\tilde{C}$ is a

constant $\left(\tilde{C} \simeq 0.3\right.$, when $\left.q_{i}=10^{-2}\right)$. Strictly, $z$ should be a discrete number and correspond to the penultimate shell, one step up from the anthropic one. In our continuum approximation, however, $z$ is a continuous variable, which we can evaluate on the surface of the anthropic shell.

Transitions will occur in all $J$ dimensions of the space. However, we see that $\kappa_{F}$ is very strongly dependent on the value of $z$, allowing us to approximate the probability density on the anthropic sphere as being primarily due to transitions from the largest of these $z$. We again consider the sphere as being divided into $2 J$ symmetric sectors. The integral that we are now required to evaluate is

$$
\left\langle f^{2}\right\rangle=\int_{0}^{1} d z \prod_{i}^{J-1} \int_{-z}^{z} d x_{i} f^{2} e^{-\tilde{C} / z^{3}} \delta\left(\sum_{i}^{J-1} x_{i}^{2}+z^{2}-1\right) .
$$

The Cartesian coordinates $x_{i}$ correspond to all of those which are orthonormal to the central axis $z$. The limits of integration, $-z$ and $z$ pick out the sector of interest. 
In appendix $\mathrm{C}$ we show that the single peak approximation for the final transition is accurate to better than $0.03 \%$ at $J=500$, and that this accuracy increases as $J$ is increased. Having justified the validity of this approximation, we will now use it to obtain analytic expressions for the peaking of probability produced by the final transition.

\subsection{Peaking From the Last Step}

Working in the single peak approximation we can use saddle points methods, as before, to estimate the level of peaking from the final transition. We begin with the integral

$$
N=\int \kappa d \Omega=2 J \int_{0}^{1} d z \prod_{i}^{J-1} \int_{-z}^{z} d x_{i} e^{-\tilde{C} / z^{3}} \delta\left(\sum_{j}^{J-1} x_{i}^{2}+z^{2}-1\right)
$$

which has its integrand extremized at

$$
z \sim\left(\frac{3 \tilde{C}}{J-2}\right)^{1 / 5}
$$

Again, the integral has most of it's support relatively close to the $z$ axis, consistent with the single peak approximation.

Once again we can calculate the expected fluxes from the integral

$$
\left\langle z^{k}\right\rangle=\frac{V_{J-2}}{N} \int_{0}^{\infty} d r\left(1-r^{2}\right)^{(k-1) / 2} r^{J-2} e^{-\tilde{C} /\left(1-r^{2}\right)^{3 / 2}},
$$

which has its saddle point given by 42 (with an $O\left(\epsilon k^{2} / J\right)$ correction), and thus

$$
\left\langle z^{k}\right\rangle \sim\left(\frac{3 \tilde{C}}{J-2}\right)^{k / 5} \sim\left(\frac{1}{J}\right)^{k / 5}
$$

This allows us to write a typical vector as

$$
q \vec{n} \sim\left(\frac{1}{J^{1 / 5}}, \frac{1}{J^{1 / 2}}, \ldots, \frac{1}{J^{1 / 2}}\right) .
$$

For $J=500$ and $q=10^{-2}$ we should then typically expect to find one set of preferred fluxes to be over 6 times greater than the others:

$$
\vec{n} \sim(29,4.5, \ldots, 4.5)
$$

These saddle point approximations are shown graphically in figure 5, where they are compared to direct numerical integrations. 


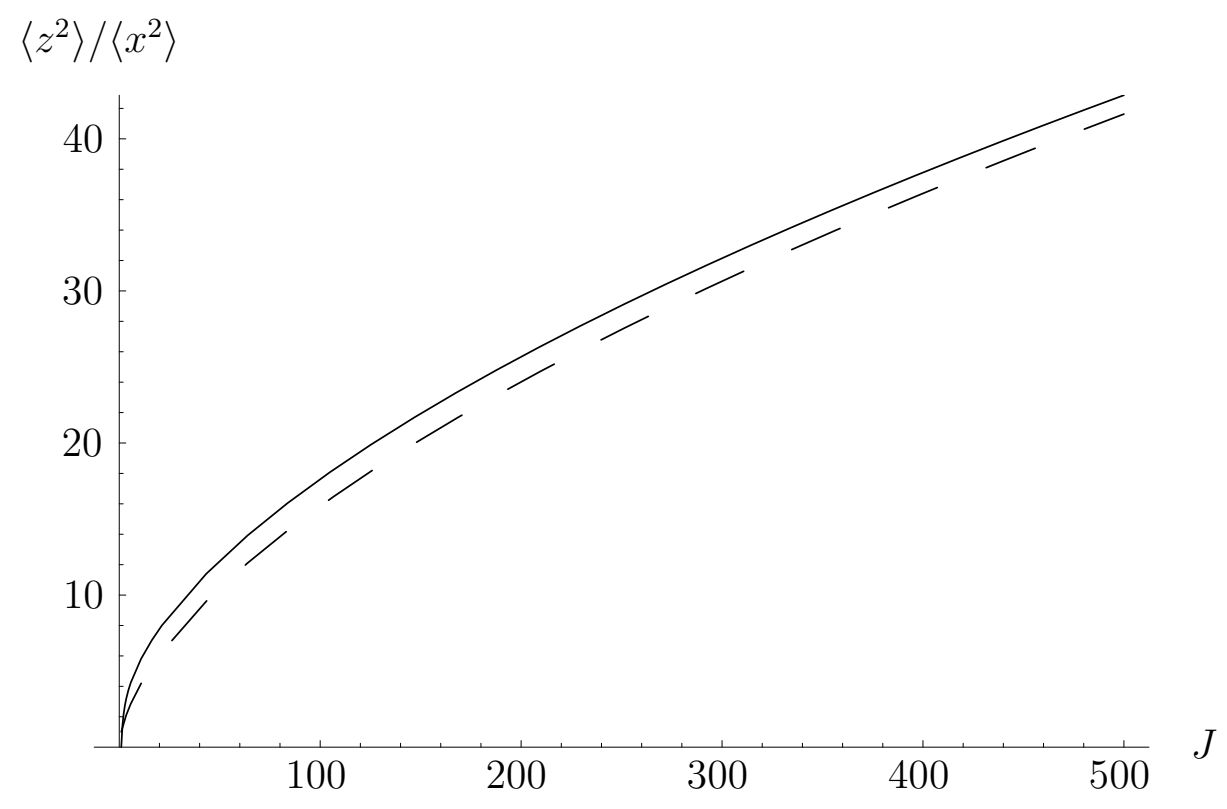

Figure 5: A plot of $\left\langle z^{2}\right\rangle /\left\langle x^{2}\right\rangle$ calculated numerically (solid line), and by saddle point approximation (dashed line). The saddle point approximation is taken from (45), and can be seen to be a reasonable approximation to the numerics at large $J$. The accuracy of the saddle point approximation increases with $J$.

The peaking found here is clearly less than that found in the previous section, and we will now show that its effects are negligible in comparison. The total tunneling rate from combining (19) and (39) is

$$
\kappa \simeq \operatorname{Exp}\left[-\frac{12}{z_{0}^{2}}-\frac{0.3}{z_{0}^{3}}\right] .
$$

It can be seen that the second term in this expression only becomes important at about $z_{0} \sim$ $1 / 40$. Before this the first term dominates, and the expression is well approximated by (19)). This critical value of $z_{0}$ can be seen to be an order of magnitude smaller than the location of the saddle points involved in either this, or the previous, section. As both of the saddlepoints are located comfortably in the region where (19) is a good approximation to the total tunneling rate, we consider the effects of this last transition to be negligible in comparison to those considered in the last section. This is supported by the relatively mild peaking found above.

\section{The Most Probable Vacua}

Having demonstrated that regions of the anthropic shell are prefentially selected by volumeweighted measures, we now turn our attention to how sharp this peaking is. We begin by finding analytic estimations for the width of the probability distributions, and then use these to obtain 
characteristic numbers for the volume of the universe that exists in some small fraction of the anthropic sphere. We then consider the issue of life-times. By imposing an anthropic limit on the minimum life-time of acceptable vacua we find that there exists a pressure favoring shorter life-times.

\subsection{Peaking About the Most Likely Point}

We will now refine our assertion that the measure on the anthropic sphere is sharply peaked, and give a demonstration of the level of this peaking. We begin our argument by considering the tunneling down from high to low energies, as discussed in section 4 , We do this by noting that near the peak the distribution is Gaussian, and then calculating the standard deviation.

We begin with the integral (30) and note that the saddle point approximation gives us not only the position of the peak, but also the width. The leading contribution to this is found to be

$$
\sigma^{2} \sim \frac{1}{8 J}
$$

Now consider the flat probability distribution on the sphere, where

$$
N_{\text {flat }}=V_{J-2} \int d z\left(1-z^{2}\right)^{(J-3) / 2},
$$

at $z \ll 1$ this can be approximated by

$$
N_{\text {flat }} \sim V_{J-2} \int d z \operatorname{Exp}\left[-J z^{2} / 2\right]
$$

Evidentally the integrand falls at least exponentially for $z \gtrsim J^{-1}$, and grows rapidly above $z \lesssim J^{-1}$. Roughly speaking, this means that whilst the probability distribution integral has most of its support around $z^{4}=24 / J$, most of the volume of the sphere lies further out, around $z=1 / J$. This is represented graphically in figure 6, where it is clear that the overlap between the probability peak and the volume peak decreases as $J$ is increased - i.e. more and more of the volume of the anthropically acceptable parts of the multiverse come from smaller and smaller fractions of the surface of the anthropic sphere.

We can then estimate what fraction, $f$, of the sphere is covered by $m \sigma$ of the probability distribution, by evaluating the volume integral (49) between the limits $\langle z\rangle-m \sigma$ and $\langle z\rangle+m \sigma$ :

$$
f=2 J \frac{V_{J-2}}{V_{J-1}} \int_{\langle z\rangle-m(8 J)^{-1 / 2}}^{\langle z\rangle+m(8 J)^{-1 / 2}} d z \operatorname{Exp}\left[-J z^{2} / 2\right] .
$$

We can bound this integral by multiplying the maximum of volume integrand (which is at $z=$ $\left.(2 K)^{1 / 4} / J^{1 / 4}+m(8 J)^{-1 / 2}\right)$ by the total range of the integral $(2 m \sigma)$. The leading contribution is given by

$$
f \sim 2 J \frac{V_{J-2}}{V_{J-1}} \frac{2 m}{J} e^{-J z^{2} / 2} \sim 2 J \frac{V_{J-2}}{V_{J-1}} \frac{2 m}{J} e^{-\frac{(24 J)^{1 / 2}}{2}} \sim 4 m \sqrt{J} e^{-\frac{(24 J)^{1 / 2}}{2}} .
$$




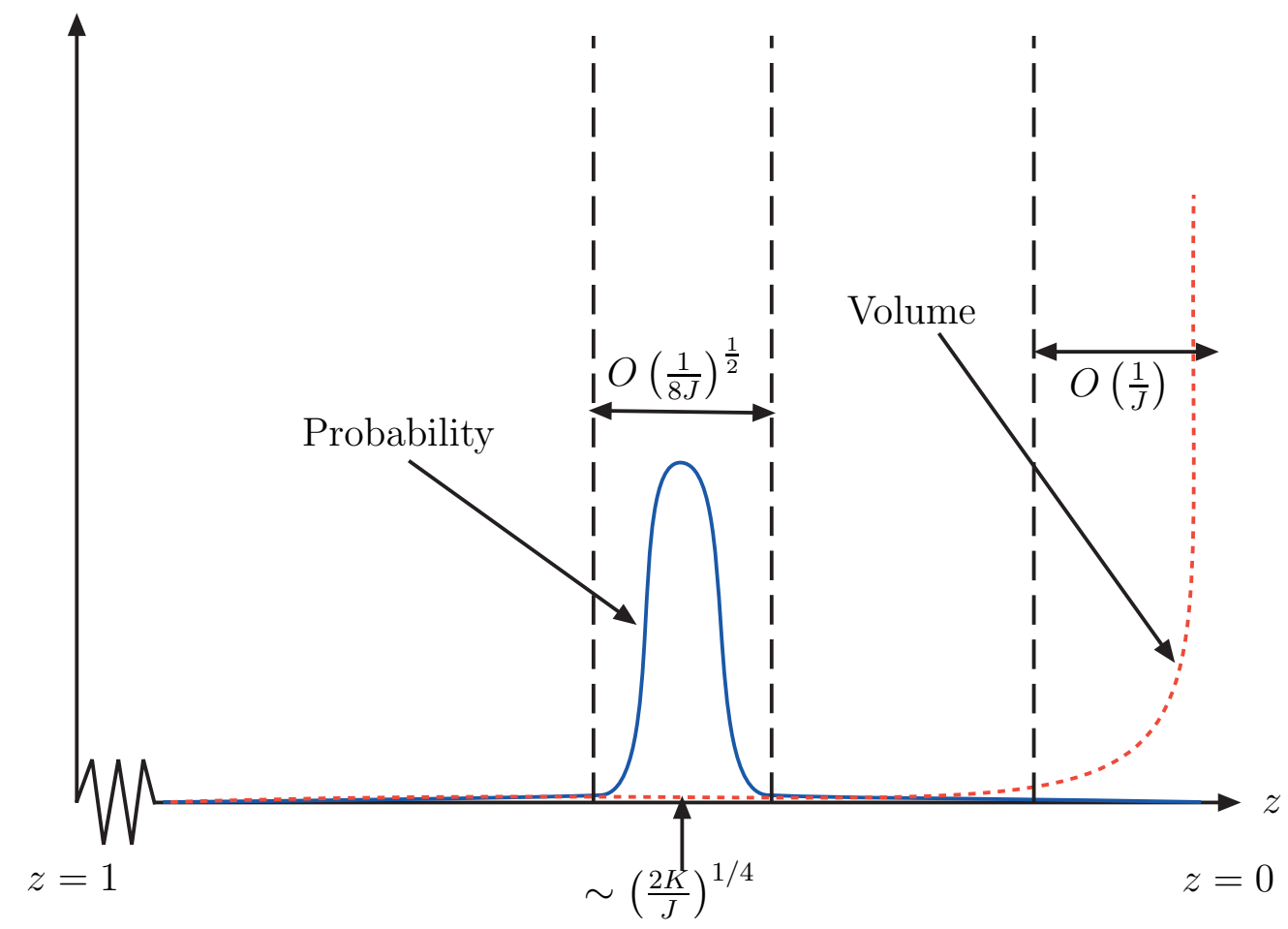

Figure 6: A sketch of the peaking of the probability measure as a function of $z$, along with the volume of the sphere. The overlap between the probability peak and the volume peak decreases as $J$ is increased.

For $J=500$ we find that $99.9999 \%$ of the volume of the universe ( 5 standard deviations) covers $\sim 10^{-23}$ of the anthropic region.

This argument can be repeated for the final transition down onto the anthropic sphere, discussed in section 5. Here the fraction of vacua on the anthropic sphere within $m \sigma$ of the peak is

$$
f \sim 2 J \frac{V_{J-2}}{V_{J-1}} \frac{2 m}{J^{7 / 10}} e^{-\frac{J^{3 / 5}}{2}} \sim 4 m J^{4 / 5} e^{-\frac{J^{3 / 5}}{2}},
$$

where $\sigma \sim J^{-7 / 10}$. For $J=500$ we then find that $99.9999 \%$ of the volume covers only $\sim 10^{-6}$ of the anthropic sphere. Evidentally, this is considerably less than that due to tunneling down from higher energies.

\subsection{Pressure Against the Life-Time Boundary}

In the preceding sections we have found the most probable vacua that have a cosmological constant in the anthropically acceptable range $0 \lesssim \Lambda \lesssim 10^{-120}$. However, for observers to appear we require not only an anthropically acceptable value of $\Lambda$, but also a vacuum that is 
long enough lived for life to evolve. In section 2.3 this was taken to be $\sim 10$ Gyr. The vacua located at the peaks of probability (found in the previous sections) do not satisfy the bound $\tau>10 \mathrm{Gyr}$, and so are unlikely to be stable enough for observers of our type to occur. If we wish to determine the probability of vacua that have both an acceptably small $\Lambda$, and an acceptably large life-time, then we must calculate probabilities only up to the boundary at which $\tau \simeq 10$ Gyr. Using our knowledge that there exists a sharp peak of probability outside of this region, we should expect to find a selection pressure pushing against the anthropic life-time barrier (so that vacua closer to the peak are favored). Here we will investigate how strong this selection pressure is, and use it to make a prediction for the half-life of acceptable vacua.

In order to establish the extent to which vacua near the life-time boundary are favored we will consider a thin region next to it. The life-time boundary is at a constant value of $z$, and we will label it by $z_{1}$. The thin region we are considering next to this boundary will extend down to a constant $z=z_{2}$, where $z_{2}<z_{1}$. The volume contained within this region can be approximated by

$$
\Delta V \simeq V_{J-2} \int_{z_{2}}^{z_{1}} e^{-\frac{J}{2} z^{2}} d z
$$

where $V_{J-2}$ is the volume of a $(J-2)$-sphere. The probability of finding a vacuum in this region can then be estimated using the formula (19), and by integrating over the thin band:

$$
\Delta P \simeq \int_{z_{2}}^{z_{1}} \kappa_{T} d \Omega \simeq \int_{z_{2}}^{z_{1}} e^{-\frac{J}{2} z^{2}-\frac{12}{z^{2}}} d z .
$$

These integrals can be performed numerically, the results of which are shown in figure 7. Here we have normalized $\Delta P$ so that $\Delta P / P \rightarrow 1$ as $z_{2} \rightarrow 0$. The volume $\Delta V$ is presented as a fraction of the volume of the unit sphere. It can be seen from the figure that the probability is pressed up against the boundary, within a fractional volume of $\sim 0.001$.

In order to determine the typical distance of an anthropically acceptable vacuum from this boundary we will find the width of strip that is required for half of the probability to be within it, and half outside of it: The half-life of the distribution. The value of $z_{2}$ for such a band is $\sim 0.9995 z_{1}$. The life-time of vacua at this value of $z$ is $\sim 12.2$ Gyr. Given the model we have been investigating, with the parameters chosen and assumptions made, we should therefore expect a typical anthropically acceptable vacuum to decay after about 2.2 billion years from the moment when the first observers appear (at 10Gyr).

One may have initially suspected that the exponential pressure up against the boundary should cause decay of vacua after a very short time. However, the exponential sensitivity of life-time on $z$ counters this pressure, and results in vacua being reasonably long lived.

\section{Discussion}

In this paper we have considered the application of volume weighted measures of eternal inflation to the Bousso-Polchinski landscape. We have found that the cosmological dynamics of volume- 


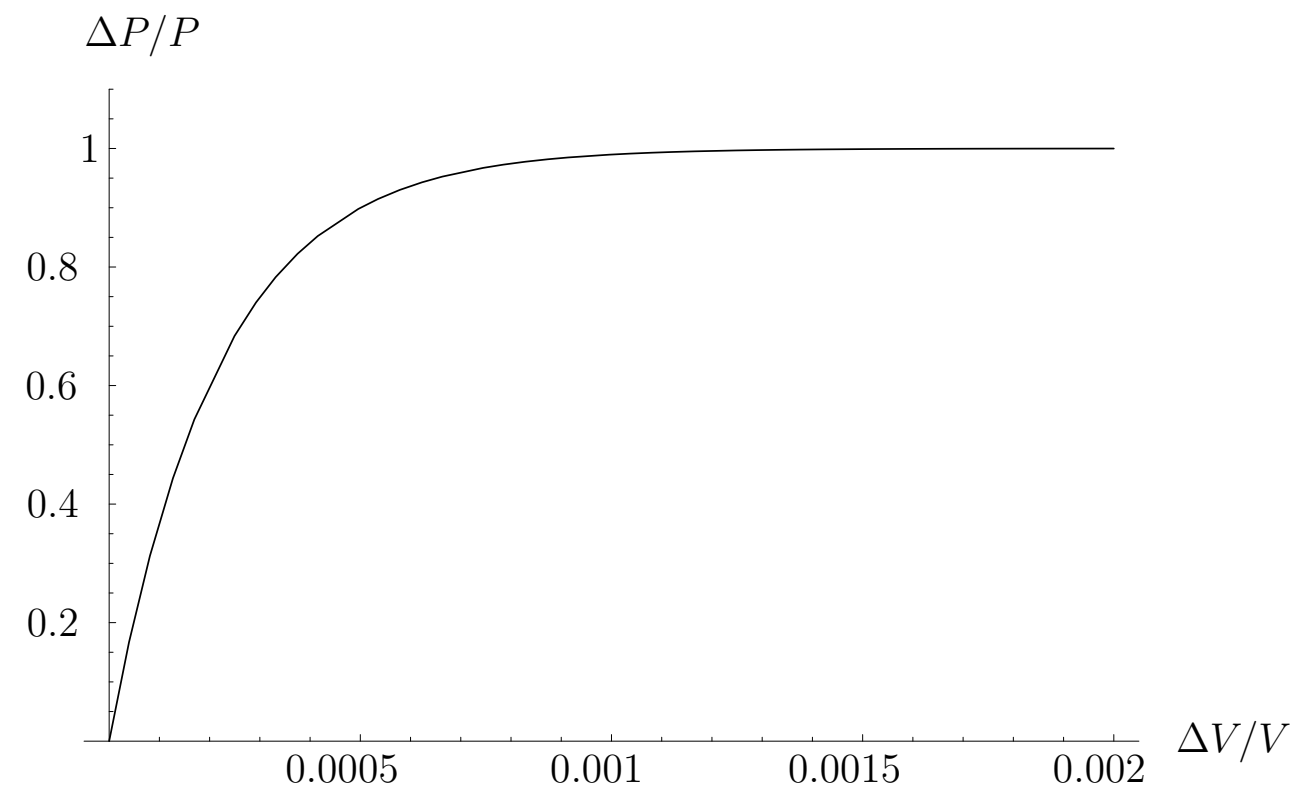

Figure 7: A plot of probability, $\Delta P$, in a thin band of volume $\Delta V$ next to the life-time boundary. Here we considered the model $J=500, q=10^{-2}$ and $\Lambda_{0}=1 / 2$.

weighting results in significant peaking of probability on the surface of the anthropic shell. The anisotropy of tunneling rates in these models leads to the selection of vacua that have one flux approximately ten times larger than the other 499 (for $J=500, q=10^{-2}$ and $\Lambda_{0}=1 / 2$ ). The peaking of probability about this most likely outcome is very sharp, with $99.9999 \%$ of the probability on the anthropic shell expected to be in a region that is only $\sim 10^{-23}$ of its surface. The preferential selection of a single large flux might suggest that warped compactifications are favored by this measure. This is in contrast to the results of Bousso and Yang [9] who find that the holographic measure [8] selects fluxes along the diagonal of the flux space, with no one flux being especially large.

Although this peaking gives us a sharp prediction for the flux configuration we should expect from volume-weighted inflation, it still allows for a satisfactory explanation of the cosmological constant problem. The reason for this is the very large number of points that are typically in the anthropic shell, $\sim 10^{489}$ for the parameters chosen above. Even an area as small as $10^{-23}$ of the anthropic shell still then contains $\sim 10^{466}$ vacua with anthropically acceptable values of the cosmological constant 7 . This is more than enough to produce the desired smooth distribution in $\Lambda$ that is required for an acceptable anthropic explanation of the problem. We do not expect the volume weighted measures of inflation to produce a staggered distribution, such as that found in the bubble counting measure of [6] for some values of parameters [7], [19].

We have also found that the most likely vacua in the anthropic shell often have a life-time too short for observers, such as ourselves, to appear. This additional anthropic pressure makes

\footnotetext{
${ }^{7}$ Other reasonable values of parameters also produce acceptably large numbers.
} 
the vacua that are sufficiently long-lived, and as close as possible to the favored points, the most likely for observers to find themselves in. Such a configuration selects vacua that are long-lived enough for observers to occur, but short-lived enough to be as close as possible to the overall peak of probability. We find that if it takes 10 billion years for observers to occur, then the most likely vacua for them to find themselves in will decay a further 2.2 billion years after this time, in our simple model. This result is dependent on the decay rates in section 2.2 being applicable to every vacuum in the anthropic shell. If anomalous vacua with longer life-times (due to e.g. super-symmetry) are identified, then these vacua would be immune to this selection pressure and may well prove to be more likely, depending on their number and flux configuration.

This apparent prediction of a doomsday might provide a plausible explanation of the "coincidence problem" of cosmology. This problem, loosely stated, asks why it should be that the energy densities observed in dark energy and matter should be comparable at the present time. If our pocket of the universe is allowed to exist forever, then a randomly distributed observer should expect to find themselves at a time when the dark energy dominates, and all other energy densities are negligible. The existence of a doomsday, a few billion years after the first observers appear, would change this picture and make it considerably more likely for a random observer to find comparable values of the dark energy and matter densities in their observable universe. A number of other solutions to this problem have also been proposed in the literature. For example, a compelling and model independent solution exists in the context of holography [20].

The results above have been calculated under the assumption that only tunneling events to nearest neighbors in the landscape are possible. However, it may be the case that multiple branes can be nucleated simultaneously, in which case we would have to be re-evaluate our results. For example, according to the results of Garriga and Megevand [15] the probability for simultaneously nucleating a stack of $m$ branes, if possible, is approximately given by $\kappa^{m}$, where $\kappa$ is the rate of nucleation of single branes. If a result of this kind can reliably be applied to the situation we have been considering here, then it will have important consequences for our results - such an event, although unlikely, would be more likely than nucleating a single brane, and then $m-1$ subsequent further branes from the lower energy vacua produced. To see this notice that the tunneling rate (19), for transitioning from the highest energy vacua to low energies, would become $\kappa_{T} \sim e^{-12 / m z_{0}^{2}}$. Here we have multiplied the numerator of the exponent by $m$, as suggested by [15], and multiplied the denominator by $m^{2}$, as the larger jumps produced by nucleating multiple branes are equivalent to rescaling $z_{0} \rightarrow m z_{0}$. A large $m$ can now be seen to decrease the exponent of $\kappa_{T}$, therefore reducing the peaking. Clearly the possibility of multiple brane nucleation events, if allowed to occur, will have a potentially important effect.

Multiple brane nucleation events could also have a second important effect, related to the minimum tunneling rate alluded to in section 2.2. If it should be the case that any number of branes can be nucleated simultaneously, then even the most unlikely events cannot occur at a rate less than $e^{-8 \pi^{2} / \Lambda}$, if they are allowed to occur at all. For vacua with sufficiently large $\Lambda$ 
this rate is not negligible, and tunneling events to distant corners of the landscape could occur with a frequency comparable to that associated with vacua that are much closer. Clearly, in such a regime the sequences of tunneling events discussed above make very little sense; instead one would expect a very nearly flat distribution of probability over the entire landscape as the highest energy vacua decay to all other vacua at approximately the same minimum rate. This is a very different picture to the one we have been focusing up in this paper. If only one brane is allowed to be nucleated at a time we have very strong peaking of probability on the anthropic sphere, if, however, any number of branes can be nucleated together then we have no peaking whatsoever. As it is this peaking that is responsible for a preference for short-lived vacua, removing it also removes this pressure. In these situations it would be the combined effect of the many unlikely transitions that would be of greatest importance.

Another simplification that we have employed in this study is to neglect the necessary period of slow-roll at the end of inflation, which is not included explicitly in the BoussoPolchinski landscape. Linde argues in [5] that volume weighted probabilities should be weighted by the amount of volume produced during the slow roll period. Such exponentially large factors could swamp the effects we have discussed here. Clearly it will be important to have a proper understanding of how to include slow-roll in this picture, in order to gain a complete understanding of how volume-weighted inflation proceeds in a more realistic landscape.

\section{Acknowledgements}

We would like to thank R. Bousso, A. Linde, K. Olum, L. Susskind and A. Vilenkin for very helpful comments and discussions. This work is supported by NSF grant PHY-0244728. TC acknowledges the support of the Lindemann trust.

\section{A Changing the Location of the High-Energy Barrier}

In section 4 the tunneling rates to the penultimate sphere, above the anthropic one, were calculated in the limit of the high-energy 'smeared out' surface being at an infinite distance from the anthropic surface. From the results of section 3 it is clear that this surface should, in fact, be at a finite distance, and so we must justify our approximation of it being at infinity. To achieve this we will take some plausible values for the actual location of this boundary, as suggested by section 3, and use them to evaluate the quantities we are interested in. We will find that the results of our calculations are relatively insensitive to the precise location of the high-energy surface, so that we are justified in approximating it to be at infinity.

Let us first consider a boundary defined by the condition $3 \Delta H=\kappa_{i \downarrow}$. This condition can be considered as an upper estimate for the high-energy surface: Vacua that meet this condition are still within the 'smeared out' region. Using the high energy tunneling rate (11) for $\kappa_{\downarrow i}$, this condition picks out a surface that can be used as an upper limit for the integral (18). This surface intersects the $z$-axis at $z \simeq 1.23$, and drops to slightly lower $z$ as you move away from the axis. 
Alternatively, we may wish to consider a high-energy boundary defined by $3 \Delta H=10 \kappa_{i \downarrow}$. Vacua that meet this condition have violated the condition to be in the smeared out region by an order of magnitude, and so can be considered to be comfortably outside of the smeared regime. This condition is then a lower estimate for the high-energy surface. Again, using (11) this condition picks out a surface which can be used as an upper limit for the integral (18). This lower estimate cuts the anthropic shell, and intersects the $z$-axis at $z \simeq 0.61$. Again, this surface drops to lower $z$ as you move away from the axis. The intersection of this surface with the anthropic shell clearly gives a different picture - now tunneling to vacua in the anthropic shell, above this surface, can be tunneled to in an unsuppressed way. Vacua below this surface must be reached in the usual way, by a downward series of suppressed jumps.

Using these two estimates for the high-energy surface we can numerically find the quantity we are most interested in: The saddle-point of the integral $\int \kappa_{\downarrow i} d \Omega$. This quantity determines the position of the peak of probability, and allows a straightforward estimate of the moments $\left\langle z^{2}\right\rangle$ and $\left\langle x^{2}\right\rangle$ (see section 4.3). The location of the saddle-point in these estimates, together with the case of a high-energy boundary at infinity, are given in the table below.

\begin{tabular}{l|ccc}
\hline $\begin{array}{l}\text { Location of } \\
\text { high-energy surface }\end{array}$ & $\begin{array}{c}\text { low } \\
\left(3 \Delta H=10 \kappa_{\downarrow i}\right)\end{array}$ & $\begin{array}{c}\text { high } \\
\left(3 \Delta H=\kappa_{\downarrow i}\right)\end{array}$ & $\begin{array}{c}\text { infinity } \\
(z \rightarrow \infty)\end{array}$ \\
\hline $\begin{array}{l}\text { Location of } \\
\text { saddle-point }\end{array}$ & $z_{0}=0.433$ & $z_{0}=0.439$ & $z_{0}=0.440$ \\
\hline
\end{tabular}

It can be seen that the location of the saddle-point is only weakly dependent on the location of the high-energy surface. The difference between our higher estimate for the 'smeared out' surface, and taking this surface to be at infinity, is only at the level of $\sim 0.2 \%$. We attribute this to the fact that most of the suppression is due to transitions at low energy, so including extra transitions at high-energy gives only a modest contribution. The difference between our lower estimate, and taking the high-energy surface to infinity, is still relatively small at the level of $\sim 1.6 \%$. This is surprisingly accurate considering that in this approximation some of the transitions are completely unsuppressed. We attribute this accuracy to the unsuppressed points near the $z$-axis contributing only a small amount to the total probability flux, due to the small amount of area near the pole compared to the large area away from it. Further from the pole, where the area is much larger, the high-energy surface is still far enough away to be well approximated by being at infinity. 


\section{B Expectation Values Due to Tunneling From High En- ergy}

To evaluate the expectation values $\left\langle z^{2}\right\rangle$ and $\left\langle x^{2}\right\rangle$, from equation (21), consider the integral

$$
\begin{aligned}
I & =\int_{0}^{\infty} d z \prod_{i}^{J-1} \int_{-z}^{z} d x_{i}\left(\sum_{i} x_{i}^{2}+z^{2}\right) e^{-\left(\sum_{i} x_{i}^{2}+z^{2}\right)} f^{2} \kappa_{T} \\
& =\int_{0}^{\infty} r^{J+1} e^{-r^{2}} d r \int_{\sigma} f^{2} \kappa_{T} d \Omega \\
& =\frac{1}{2} \Gamma\left(\frac{J+2}{2}\right)\left\langle f^{2}\right\rangle,
\end{aligned}
$$

where $f=f\left(x_{i}, z\right)$ is any function of $x_{i}$ and $z$ on the surface of the sphere. In moving from the first to second line we have simply transformed to a set of hyper-spherical polar coordinates, where $r \equiv \sum_{i} x_{i}^{2}+z^{2}$. In order to evaluate this second line, and obtain the third, we have had to be careful in how we have defined $\kappa_{T}$; specifically, we have required that $\kappa_{T}=\kappa_{T}(\Omega)$. This may at first seem contrary to (19), but it should be remembered that we are only interested in the value of $\kappa_{T}$ on one particular surface - that of the anthropic shell. We can then re-write (19) as

$$
\kappa_{T} \sim e^{-12 / \hat{z}^{2}},
$$

where $\hat{z}=\hat{z}(\Omega)$ is the value of $z$ on the unit sphere at the point described by the polar coordinates $\Omega$. For points not contained on the unit sphere, $\kappa_{T}$ is obtained by connecting the point to the origin with a radial vector and reading off the value of $\hat{z}$ as this vector crosses the surface of the unit sphere. Similar triangles then give

$$
\frac{\sum_{i} x_{i}^{2}+z^{2}}{1}=\frac{z^{2}}{\hat{z}^{2}}
$$

or

$$
\kappa_{T} \sim e^{-12\left(\sum_{i} x_{i}^{2}+z^{2}\right) / z^{2}} .
$$

Similarly, we can re-write $x^{2}$ as

$$
\frac{\sum_{i} x_{i}^{2}+z^{2}}{1}=\frac{x^{2}}{\hat{x}^{2}}
$$

where $\hat{x}=x(\Omega)$ is the corresponding value of $x$ at the point a suitable radial vector crosses the anthropic shell. With these new expressions for $z^{2}$ and $x^{2}$, the integral (55) can be written as

$$
\left\langle\hat{f}^{2}\right\rangle=\frac{2}{\Gamma\left(\frac{J+2}{2}\right)} \int_{0}^{\infty} d z \prod_{i}^{J-1} \int_{-z}^{z} d x_{i} f^{2} e^{-\left(\sum_{i} x_{i}^{2}+z^{2}\right)\left(1+12 z^{-2}\right)} .
$$

Taking $f=z$ the integrals over the coordinates $x_{i}$ can all be performed exactly, giving the moment $\left\langle z^{2}\right\rangle$ as

$$
\left\langle\hat{z}^{2}\right\rangle=\frac{2}{\Gamma\left(\frac{J+2}{2}\right)} \int_{0}^{\infty} d z z^{2}\left[\frac{\sqrt{\pi} z}{\sqrt{12+z^{2}}} E\left(\sqrt{12+z^{2}}\right)\right]^{J-1} e^{-\left(12+z^{2}\right)}
$$


where $E(p)$ is the integral of a Gaussian distribution, or error function, given by $2 \pi^{-1 / 2} \int_{0}^{p} e^{-q^{2}} d q$. This analysis has reduced $\left\langle z^{2}\right\rangle$ from a $J$ dimensional integral, which is potentially very difficult to solve, to a one dimensional integral that can be solved by saddle point approximation, or by a simple numerical calculation.

We can proceed in a similar fashion to obtain an expression for $\left\langle x^{2}\right\rangle$, which can also be expressed as a one-dimensional integral. Such an analysis gives

$$
\begin{aligned}
\left\langle\hat{x}^{2}\right\rangle & =\frac{2}{\Gamma\left(\frac{J+2}{2}\right)} \int_{0}^{\infty} d z \prod_{i}^{J-1} \int_{-z}^{z} d x_{i} x^{2} e^{-\left(\sum_{i} x_{i}^{2}+z^{2}\right)\left(1+12 z^{-2}\right)} \\
= & \frac{2}{\Gamma\left(\frac{J+2}{2}\right)} \int_{0}^{\infty} d z \int_{-z}^{z} d x x^{2}\left[\frac{\sqrt{\pi} z}{\sqrt{12+z^{2}}} E\left(\sqrt{12+z^{2}}\right)\right]^{J-2} e^{-\left(x^{2}+z^{2}\right)\left(1+12 z^{-2}\right)} \\
= & \frac{2}{\Gamma\left(\frac{J+2}{2}\right)} \int_{0}^{\infty} d z z^{2}\left(\frac{1}{2\left(12+z^{2}\right)}-\frac{e^{-\left(12+z^{2}\right)}}{\sqrt{\pi} \sqrt{12+z^{2}} E\left(\sqrt{12+z^{2}}\right)}\right) \\
& \times\left[\frac{\sqrt{\pi} z}{\sqrt{12+z^{2}}} E\left(\sqrt{12+z^{2}}\right)\right]^{J-1} e^{-\left(12+z^{2}\right)} .
\end{aligned}
$$

Going from the first to the second line, we have integrated over all of the $x_{i}$ dimensions, except for $x$ (that is, the particular $x_{i}$ we are calculating the expectation value of). Going from the second to third lines we then integrate over $x$.

\section{The Single Peak Approximation for the Final Transi- tion}

The integral (40) is not trivial to evaluate, and so we will briefly consider a toy model in which the calculations are significantly simpler to perform. This model will allow us to fix ideas and explicitly investigate approximations which will be of direct use in solving (40). The toy model we propose is one in which the rate of transitions goes as

$$
\kappa_{\text {toy }} \sim e^{-\hat{C} / z^{2}}
$$

where $\hat{C}$ is a constant. This rate is clearly the same as (19), with a different numerical factor in the exponent. We already know such models can be solved exactly, from the preceding section. We will use this knowledge to justify the single peak approximation for the tunneling rate (39).

In order to show that the single peak approximation is valid for (39), we will proceed as follows. First we will investigate this approximation in our toy model. Having shown the extent to which this approximation is valid in our toy model, we will argue that it should be a significantly better approximation in a more realistic model. We will then show the results

of numerical calculations of the more realistic model at low dimensions. This will support our 


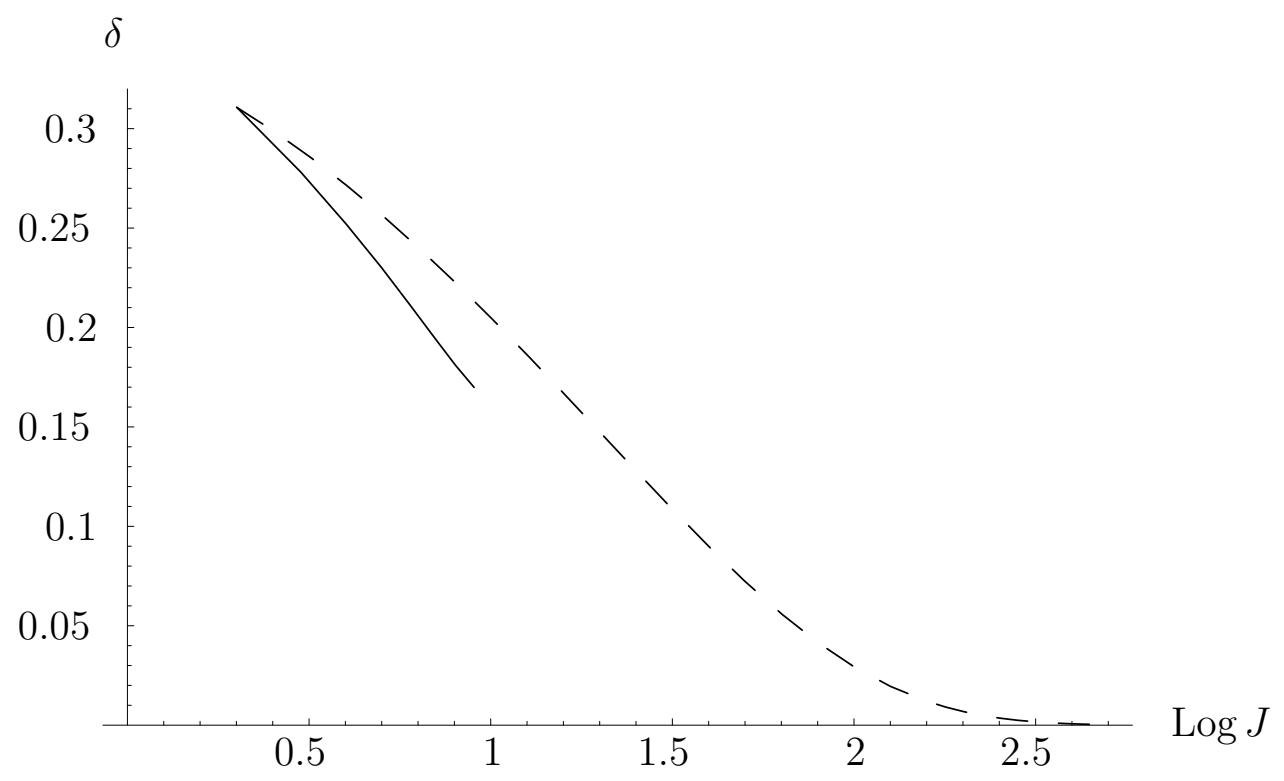

Figure 8: A plot of $\delta$ as a function of dimensionality of the space, $J$, in the toy model (dashed line) and in a more realistic model (solid line). The value of $\hat{C}$, in the toy model is taken to be 0.7 , and the value of $\tilde{C}$, in the more realistic model is 0.3 .

previous argument and, when extended to larger dimensions, should provide credible evidence that the single peak approximation is good.

For our toy model, the single peak approximation yields the results as for (19), with 200 replaced by $\hat{C}$. We now plot $\delta$ for the present case as a function of $J$ in figure 8, It can be seen from the figure that the error, $\delta$, can be as large as $\sim 0.3$ at low dimensionality $(J \rightarrow 2)$. At larger dimensionality, however, the value of $\delta$ can be seen to drop off, and for $J=500$ can be seen to be less than $0.03 \%$. We expect that this is due to the fact that at higher dimensionality the boundaries of the domain of integration of the actual region of interest extends to lower and lower values of $z$. This can be seen straight-forwardly from the expression for the diagonal (that is, the place furthest from all axes where the values of all coordinates are equal):

$$
\sum_{i}^{J} x_{i}^{2}=1 \quad \Rightarrow \quad x_{i} \sim \frac{1}{\sqrt{J}} .
$$

As this region extends to lower $z$, it covers more of the region that is integrated over in the single peak approximation, making the approximation increasingly more accurate as more dimensions are added.

Having established that the single peak approximation in our toy model is accurate to better than $0.03 \%$, when $J=500$, we can begin to consider the accuracy of this approximation in more realistic models. We expect that the single peak approximation should be more accurate in the more realistic models. The reason for this is that the rate of transitions is a steeper 
function of $z\left(e^{-1 / z^{3}}\right.$ instead of $\left.e^{-1 / z^{2}}\right)$. With this in mind we should expect the extra area integrated over in the single peak approximation to make even less of a contribution, as this extra area is at low $z$.

The calculation to be performed in the more realistic model is particularly cumbersome at large $J$, as we do not have the pleasant reduction to a one-dimensional integral that was exhibited in the toy model. For low-dimensionality, however, numerical evaluations can be performed. The recipe in this case is similar to that of the toy model. Consider the integral

$$
\begin{aligned}
I & =\int_{0}^{\infty} d z \prod_{i}^{J-1} \int_{-z}^{z} d x_{i}\left(\sum_{i} x_{i}^{2}+z^{2}\right) f^{2} e^{-\left(\sum_{i} x_{i}^{2}+z^{2}\right)^{3 / 2}} \kappa_{F} \\
& =\int_{0}^{\infty} r^{J+1} e^{-r^{3}} d r \int_{\sigma} f^{2} \kappa_{F} d \Omega \\
& =\frac{1}{3} \Gamma\left(\frac{J+2}{3}\right)\left\langle f^{2}\right\rangle .
\end{aligned}
$$

Now, by the same method as before, we write the transition rate as a function of angular coordinates

$$
\kappa_{F} \sim e^{-\tilde{C}\left(\sum_{i} x_{i}^{2}+z^{2}\right)^{3 / 2} / z^{3}}
$$

and

$$
\frac{\sum_{i} x_{i}^{2}+z^{2}}{1}=\frac{f^{2}}{\hat{f}^{2}}
$$

so that (60) gives

$$
\left\langle\hat{f}^{2}\right\rangle=\frac{3}{\Gamma\left(\frac{J+2}{3}\right)} \int_{0}^{\infty} d z \prod_{i}^{J-1} \int_{-z}^{z} d x_{i} f^{2} e^{-\left(\sum_{i} x_{i}^{2}+z^{2}\right)^{3 / 2}\left(1+\tilde{C} z^{-3}\right)}
$$

In the single peak approximation this integral becomes

$$
\left\langle\bar{f}^{2}\right\rangle=\frac{3}{\Gamma\left(\frac{J+2}{3}\right)} \int_{0}^{\infty} d z \prod_{i}^{J-1} \int_{-\infty}^{\infty} d x_{i} f^{2} e^{-\left(\sum_{i} x_{i}^{2}+z^{2}\right)^{3 / 2}\left(1+\tilde{C} z^{-3}\right)} .
$$

This last integral can be written in a significantly simpler way in terms of a set of hypercylindrical coordinates (which has been the reason for this whole subsection). Here, however, this simplification is not particularly beneficial as in order to evaluate $\delta$ we must also analyze the more complicated integral (61), over the actual region of interest.

The results of numerically integrating (61) and (62) are also shown in figure 8 . Here $\delta$ is defined in the same way as in the toy model, and we have set $\tilde{C}=0.3$. The value of $\hat{C}$, for the toy model, was taken as 0.7 in order to match the two models at $J=2$ (the lowest dimensionality for which $\delta$ is well defined). It can be seen that the magnitude of $\delta$ in this more realistic model is indeed smaller than in the toy model, as conjectured earlier. Although we 
do not have reliable estimates of $\delta$ at large $J$ in the more realistic model (due to the difficulty of numerically integrating over a large number of dimensions), we argue by analogy that we should expect $\delta$ to continue dropping as $J$ is increased.

This concludes our argument in favor of the validity of the single peak approximation. We have shown that there exists a toy model in which $\left\langle f^{2}\right\rangle$ can be evaluated in a more straightforward fashion. In this toy model we have shown that the single peak approximation is accurate to $\sim 0.03 \%$ at $J=500$, and that this accuracy increases as $J$ is increased. We have then argued that in our more realistic model the single peak approximation should be more accurate than in the toy model. Numerical investigations at low dimensionality indicate that this expectation is correct, and comparison with the toy model leads us to expect that the single peak approximation in more realistic models should indeed be a good approximation at large $J$.

\section{References}

[1] For a review see M. Douglas and S. Kachru, "Flux Compactification," arXiv:hep-th/0610102.

[2] R. Bousso and J. Polchinski, "Quantization of Four-Form Fluxes and Dynamical Neutralisation of the Cosmological Constant," JHEP 0006, 006 (2000) arXiv:hep-th/0004134.

[3] T. Clifton, A. Linde and N. Sivanandam, "Islands in the Landscape," JHEP 0702, 024 (2007) arXiv:hep-th/0701083.

[4] A. Linde, "Eternally Existing Self-Reproducing Chaotic Inflationary Universe," Phys. Lett. B175, 395 (1986).

[5] A. Linde, "Towards a Gauge Invariant Volume-Weighted Probability Measure for Eternal Inflation," arXiv:0705.1160.

[6] J. Garriga, D. Schwartz-Perlov, A. Vilenkin and S. Winitzki, "Probabilities in the Inflationary Multiverse," JCAP 0601, 017 (2006) arXiv:hep-th/0509184.

[7] D. Schwartz-Perlov and A. Vilenkin, "Probabilities in the Bousso-Polchinski Multiverse," JCAP 0606, 010 (2006) arXiv:hep-th/0601162.

[8] R. Bousso, "Holographic Probabilities in Eternal Inflation," Phys. Rev. Lett. 97, 191302 (2006) arXiv:hep-th/0605263.

[9] R. Bousso and I.-S. Yang, "Landscape Predictions from Cosmological Vacuum Selection," arXiv:hep-th/0703206.

[10] J. Garriga and A. Vilenkin, "Recycling Universe," Phys. Rev. D 57, 2230 (1998) arXiv:astro-ph/9707292. 
[11] A. Linde, "Sinks in the Landscape, Boltzmann Brains, and the Cosmological Constant Problem," JCAP 0701, 022 (2007) arXiv:hep-th/0611043].

[12] S. Coleman and F. De Luccia, "Gravitational Effects on and of Vacuum Decay," Phys. Rev. D 21, 3305 (1980).

[13] S. Parke, "Gravity and the Decay of False Vacuum," Phys. Lett. 121B, 313 (1983).

[14] J. Brown and C. Teitelboim, "Dynamical Neutralization of the Cosmological Constant," Phys. Lett B195, 177 (1987).

[15] J. Garriga and A. Megevand, "Coincident Brane Nucleation and Neutralisation of $\Lambda$," Phys. Rev. D 69, 083510 (2004) arXiv:hep-th/0310211.

[16] S. Kachru, R. Kallosh, A. Linde and S. Trivedi, "De Sitter Vacua in String Theory," Phys. Rev. D 68, 046005 (2003) arXiv:hep-th/0301240.

[17] A. Linde, "The Inflationary Universe," Rept. Prog. Phys. 47, 925 (1984), T. Banks, "T C P, Quantum Gravity, the Cosmological Constant and All That..," Nucl. Phys. B 249, 332 (1985), S. Weinberg, "Anthropic Bound on the Cosmological Constant," Phys. Rev. Lett. 59, 2607 (1987).

[18] A. Linde, "Inflation and Quantum Cosmology," Print-86-0888 (June 1986), in 300 Years of Gravitation, ed. by S. Hawking and W. Israel, Cambridge University Press, Cambridge (1987).

[19] K. Olum and D. Schwartz-Perlov, "Anthropic Prediction in a Large Toy Landscape," arXiv:0702.2562].

[20] R. Bousso, R. Harnik, G. Kribs and G. Perez, "Predicting the Cosmological Constant from the Causal Entropic Principle," arXiv:hep-th/0702115]. 